\title{
Land Resilience and Tail Dependence among Crop Yield Distributions
}

\author{
Xiaodong Du, David A. Hennessy, and Hongli Feng
}

Working Paper 15-WP 556

April 2015

\author{
Center for Agricultural and Rural Development \\ lowa State University \\ Ames, lowa 50011-1070 \\ www.card.iastate.edu
}

Xiaodong Du is Assistant Professor of Agricultural and Applied Economics, University of WisconsinMadison.E-mail: xdu23@wisc.edu

David A. Hennessy is Professor of Economics at the Department of Economics \& Center for Agricultural and Rural Development, lowa State University. E-mail: hennessy@iastate.edu.

Hongli Feng is Adjunct Associate Professor of Economic, Iowa State University. E-mail:

hfeng@iastate.edu.

For questions or comments about the contents of this paper, please contact David A. Hennessy, 578C Heady Hall, Dept. of Economics, lowa State University, Ames IA 50011-1070. hennessy@iastate.edu.

Iowa State University does not discriminate on the basis of race, color, age, ethnicity, religion, national origin, pregnancy, sexual orientation, gender identity, genetic information, sex, marital status, disability, or status as a U.S. veteran. Inquiries can be directed to the Interim Assistant Director of Equal Opportunity and Compliance, 3280 Beardshear Hall, (515) 294-7612. 


\title{
Land Resilience and Tail Dependence among Crop Yield Distributions
}

Xiaodong Du

Dept. of Ag \& Applied Economics University of Wisconsin-Madison xdu23@wisc.edu
David A. Hennessy

Dept. of Economics

Iowa State University

hennessy@iastate.edu

\author{
Hongli Feng \\ Dept. of Economics \\ Iowa State University \\ hfeng@iastate.edu
}

\begin{abstract}
Rate setting procedures for United States crop yield and revenue insurance contracts employ methods that presume correlations to be state invariant. Whether this is true matters. If yield-yield correlations strengthen when crops are subject to widespread stress, then diversification opportunities for private insurers weaken when most needed, and an insurer's portfolio of retained business may not be as diversified as standard statistics would suggest. For government outlays, such tail dependence will increase the transactions and political costs of reallocations from the general fund. In this paper we propose a simple model of yield correlations according to interactions between a weather outcome and a land unit's yield resilience to adverse shocks, as might be measured by the United States Soil Conservation Service's land capability classification. Our model shows that yield-yield tail dependence is to be expected and, furthermore, should take a particular form. In better growing regions, yield correlations across units should be stronger in right tails than in left tails, whereas in marginal growing regions the reverse should apply. Using USDA Risk Management Agency unit level data and a variety of statistics, we find strong evidence in favor of this land yield resilience hypothesis. Our findings call into question the appropriateness of current USDA rate-setting methodologies, which posit constant state-conditional ordinal correlations by implicitly assuming that yields can be represented by a Gaussian copula. A goodness-of-fit test rejects the standard Gaussian copula model, implying that existing RMA rate-setting methods are deficient.
\end{abstract}

Keywords: actuarial fairness; crop insurance; Gaussian copula; geography of yield distributions; reinsurance; systemic risk.

JEL Codes: G12, Q18, C1. 
Diversification can be an important risk management strategy, but its effectiveness relies on the degree of orthogonality among assumed risks. If an entity is exposed to multiple independent risks, then risk sharing can effectively reduce overall risk exposure; whereas if multiple risks are positively correlated with a strong common systemic component, then pooling is only partially effective. The nature of partial correlation matters greatly. Standard financial theory, as in the workhorse Capital Asset Pricing and generalized Factor Pricing models, has presumed that correlations are state invariant. However, the global financial crisis of circa 2008 has shown security market models based on this assumption to be wanting in times of severe financial stress. Among the approaches brought into question is the popular Gaussian copula methodology (Li 2000).

Crop insurance rate setting typically involves the modeling of multiple risks. One approach to doing so is an algorithm introduced by Iman and Conover (1982). It has become a central plank in United States crop insurance rate setting methodology (Coble et al. 2010) and is embedded in popular risk management software such as @RISK by Palisade Corporation. However, as pointed out in Mildenhall (2005), underlying this approach is the Gaussian copula superstructure on marginal interactions.

A copula is a function that maps marginal distributions into a multivariate distribution. In doing so, the copula imposes some structure on the resultant multivariate distribution. Although the marginal distributions are not restricted in any way, interactions among marginals will be constrained by the copula choice. A great virtue of the multivariate normal (Gaussian) distribution is its parameterized second-order flexibility. While the Gaussian copula does not fix cardinal measures of correlation, it severely constrains ordinal (i.e., rank) correlations. Gaussian methodology is appealing if one is not concerned about higher moments and if one is prepared to accept these correlation constraints. Copulas flexible enough to capture at least some tail dependence, such as $t$ - and mixture-copulas, are found to fit the unit level yield data better than 
the Gaussian copula (Goodwin and Hungerford 2015). Similarly when modeling changes in housing prices in the interval surrounding the financial crisis of 2008, the Gaussian copula was found to be inappropriate as it imposes constant dependence interactions even for extreme events (Zimmer 2012). Zimmer (2014) has shown that commodity price-price correlations strengthen when prices decline.

There are several reasons why one should care about how crop insurance rates are modeled. One regards mispricing that could arise from erroneously assessing systemic risk levels. When compared with financial markets, systemic risk is harder to estimate for crop yields because data are annual rather than daily. Miscalculation can have severe consequences. Crop insurance markets have had a checkered history throughout the world, in large part because of adverse selection and moral hazard issues (Goodwin 2001; Glauber 2013; Just, Calvin and Quiggin 1999). Absent compulsion, history imparts that significant participation requires subsidies and/or fair premiums for each land unit. For unit-specific yield insurance contracts, failure to account for tail dependence does not necessarily mean that rates are significantly biased, but does suggest that entities holding portfolios of such contracts do not fully appreciate value at risk. Crop insurers and the federal government in the United States hold significant contract portfolios. For revenue, whole farm and area contracts, failure to account for tail dependence does suggest a bias in rates, increasing adverse selection concerns. Consider a whole farm product. As payoffs are convex in yield, if correlations among unit yields strengthen as crops become more stressed (i.e., assume more systemic risk than normal years), then diversification offsets will likely fail when most needed.

A second concern regards the costs of managing systemic risk, even if calculated correctly. The book of United States crop yield risks, though very large, is not particularly large when compared with risks assumed elsewhere in global reinsurance markets. Nor is it large when compared with the federal budget as a whole. However, the United States standard reinsurance 
agreement requires that crop insurance marketing companies retain certain risks on their books. ${ }^{1}$ For its part, the federal government chooses to act as reinsurer for the rest. A government's finances are compartmentalized, in part to ensure that the legislative branch of government retains the power of the purse across the political arena. Although contractual commitments will be met, there are political and transactions costs to doing so, as bureaucrats and legislators horse trade over accommodating budgetary constraints. More systemic yield risk increases such costs by increasing the severity of the budgetary problem countenanced.

The intents of this article are three-fold. Firstly, we will take a conceptual approach to reason why state-dependent correlation structures are to be expected. Specifically, in regard to crop yield-yield correlations we will develop a model grounded in the view that most productive cropland has few limitations, such that yields should be resilient to adverse weather shocks, whereas yields on more limited soils will prove sensitive to weather stressors. Given local heterogeneities in land productivity, our land yield resilience theory suggests that yield correlations should strengthen in better growing areas when growing conditions are good, while yield correlations should strengthen in marginal growing areas when growing conditions are poor.

Our second intent is to scrutinize historical disaggregated (at the unit level) yield records from the Risk Management Agency (RMA) branch of the United States Department of Agriculture (USDA) to shed light on whether the land yield resilience model's (LYRM) hypothesis on correlation structures is consistent with empirical evidence. We use a variety of statistics to check for state-varying systemic risk and find robust adherence to the hypothesis. Our third intent is to establish whether or not the Gaussian copula, with its state-invariant ordinal correlation structures, provides a good fit to the RMA yield record data. We find that a goodness-of-fit test rejects the standard Gaussian copula model.

\footnotetext{
${ }^{1}$ See the Assigned Risk Retention Clause on p. 14 in the 2014 Standard Reinsurance Agreement, http://www.rma.usda.gov/pubs/ra/sraarchives/14sra.pdf
} 
Our work is most closely related to that of Goodwin and Hungerford (2015), who question whether the Gaussian copula that the RMA implicitly assumes is appropriate. Goodwin and Hungerford do so by fitting alternative copulas to detrended unit-level and county-level yield data. They find evidence in favor of flexible vine copulas over the Gaussian and other copula structures. They do not specifically address tail dependence issues, do not provide a conceptual framework to rationalize why state-invariant correlation structures may be problematic, and do not present a normative theory for the nature of state-dependent correlations structures in the tails.

Our resilience model is distinct from, but entirely consistent with recent work by Du, Hennessy and Yu (2012) and Du et al. (2015). These authors stress land fertility, be it soilderived or through fertilizer application, and also weather endowments as determinants of asymmetries in the shape of univariate yield distributions. In this way, they provide a geographic framework in which to view crop yield distributions. They find evidence to indicate that better quality land decreases yield skewness, but do not address yield-yield correlation structures. Du, Hennessy and Feng (2014) also consider the role of natural resource endowments on crop insurance market outcomes. They show that a region's natural resource endowments together with systemic yield and price-yield correlations should affect preferences for crop insurance forms (revenue or yield) and coverage levels, but do not ask how natural resource endowments can affect a region's systemic risk attributes.

The remains of this paper are laid out as follows. After presenting some notions of dependence, we provide a simple relation between underlying growing conditions (which may be read as weather and/or land quality attributes) and production outcomes for heterogeneous units of land in a region. We then reason how the interaction between growing condition inputs and yield outputs should affect correlation structures for yields across those heterogeneous land units in a region. Summary statistics on correlations among disaggregated yield data are 
provided, after which we subject the data to a battery of assessments to discern how yield correlations vary by a region's land quality and by overall growing conditions. We also explicitly test for how well the Gaussian copula fits data. The work concludes with a discussion on findings and suggestions for future inquiry.

\section{Conceptual Model}

Tail dependence describes the monotone relationship between two continuous random variables. Left-tail decreasing (LTD) and right-tail increasing (RTI) orders are a widely used pair of stochastic orders that inform on any tail dependence. Details on these orders can be found in Berghaus and Bücher (2014), chapter 2 in Joe (2015) or pp. 155-159 in Nelsen (1999).

Throughout we use $P(A)$ to denote the probability of an outcome in set $A, P\left(A_{1} \cap A_{2}\right)$ to denote the probability of an outcome in both sets $A_{1}$ and $A_{2}$, and write $P\left(A_{1} \mid A_{2}\right)=P\left(A_{1} \cap A_{2}\right) / P\left(A_{2}\right)$. For two random variables, $x_{1}$ and $x_{2}, x_{2}$ is LTD in $x_{1}$ or $\operatorname{LTD}\left(x_{2} \mid x_{1}\right)$, whenever

$$
P\left[x_{2} \leq \hat{x}_{2} \mid x_{1} \leq \hat{x}_{1}^{a}\right] \geq P\left[x_{2} \leq \hat{x}_{2} \mid x_{1} \leq \hat{x}_{1}^{b}\right] \quad \forall \hat{x}_{1}^{b} \geq \hat{x}_{1}^{a}, \quad \forall \hat{x}_{2} .
$$

Similarly $x_{2}$ is RTI in $x_{1}$, or $\operatorname{RTI}\left(x_{2} \mid x_{1}\right)$, whenever

(2) $P\left[x_{2} \geq \hat{x}_{2} \mid x_{1} \geq \hat{x}_{1}^{a}\right] \leq P\left[x_{2} \geq \hat{x}_{2} \mid x_{1} \geq \hat{x}_{1}^{b}\right] \quad \forall \hat{x}_{1}^{b} \geq \hat{x}_{1}^{a}, \quad \forall \hat{x}_{2}$.

Were data to conform with these properties, then the Gaussian copula would be problematic. This is because Aas (2004) has shown that when the $\hat{x}_{2}$ is sufficiently large or, when it is sufficiently small, then the Gaussian copula will always exhibit zero conditional correlation for extreme values of $\hat{x}_{1}$, and so cannot encompass tail dependence in the senses of $\operatorname{LTD}\left(x_{2} \mid x_{1}\right)$ and $\operatorname{RTI}\left(x_{2} \mid x_{1}\right)$. We are interested in these monotonicity conditions so as to capture any tendency for the conditional distribution of $x_{2}$ to shift as tail values of $x_{1}$ are realized. Both conditions are intended to discern tail dependence with (1) and (2) focusing, respectively, on the left and right tails. The LTD and RTI relations are not equivalent as neither assert anything 
about realizations in the sets $\left\{\left(x_{1}, x_{2}\right): x_{1}>\hat{x}_{1}, x_{2} \leq \hat{x}_{2}\right\}$ and $\left\{\left(x_{1}, x_{2}\right): x_{1} \leq \hat{x}_{1}, x_{2}>\hat{x}_{2}\right\}$.

Example 1: Let $u\left(\hat{x}_{1}\right)=P\left[x_{1} \leq \hat{x}_{1}\right]$ and $v\left(\hat{x}_{2}\right)=P\left[x_{2} \leq \hat{x}_{2}\right]$. As illustration, consider the FarlieGumbel-Morgenstern copula, (i.e., of form $C(u, v)=u v+\psi u v(1-u)(1-v))$ so that $\bar{C}(u, v)=1-v-u+u v+\psi u v(1-u)(1-v) .^{2}$ Then

$$
\begin{aligned}
& \frac{d P\left[x_{2} \leq \hat{x}_{2} \mid x_{1} \leq \hat{x}_{1}^{a}\right]}{d \hat{x}_{1}}=\frac{d[C(u, v) / u]}{d u} \frac{d u}{d \hat{x}_{1}}=-\psi v(1-v) \frac{d u}{d \hat{x}_{1}} \stackrel{\text { sign }}{=}-\psi ; \\
& \frac{d P\left[x_{2} \geq \hat{x}_{2} \mid x_{1} \geq \hat{x}_{1}^{a}\right]}{d \hat{x}_{1}}=\frac{d[\bar{C}(u, v) /(1-u)]}{d u} \frac{d u}{d \hat{x}_{1}}=\psi v(1-v) \frac{d u}{d \hat{x}_{1}} \stackrel{\text { sign }}{=} \psi .
\end{aligned}
$$

In this case, both conditions are satisfied if and only if $\psi \geq 0$. See Exercise 5.35, page 168, in Nelsen (1999) for simple counterexamples showing that the two conditions are not synonymous.

Two other notions of tail dependence are (p. 26 in Trivedi and Zimmer 2005):

$$
\lambda_{L} \equiv \lim _{v \downarrow 0} \frac{P\left[\left\{x_{1} \leq q_{v}\right\} \cap\left\{x_{2} \leq q_{v}\right\}\right]}{v} ; \quad \lambda_{U} \equiv \lim _{v \uparrow 1} \frac{P\left[\left\{x_{1} \geq q_{v}\right\} \cap\left\{x_{2} \geq q_{v}\right\}\right]}{1-v} ;
$$

where $q_{v}=$ quantile $v$. Here, notation $\{\cdot\}$ is used to indicate a condition in the event that notation might otherwise be ambiguous. Both $\lambda_{L}$ and $\lambda_{U}$ have value 0 for any Gaussian copula.

\section{Land resilience and yield-yield correlations}

Abundant evidence exists that soil quality, when measured in regard to average productivity, determines yield risk (e.g., Calviño and Sadras 1999; Williams et al. 2008). For example, the USDA Soil Conservation Service categorizes land by capability classes—Class I type land has few limitations that restrict use, and higher classes have increasingly severe limitations. Among the limitations are shallow and/or panned soils, as well as physical and chemical properties that limit root penetration and water retention capacity. Although a central purpose of the classification has been to support approaches that discourage soil erosion (Helms 1992), it is

\footnotetext{
${ }^{2}$ See Theorem 5.2.5, page 156, in Nelsen (1999).
} 
widely used to measure yield productivity. Logic based on the LeChatelier principle (p. 47 in Varian 1992) suggests that better, or less constrained, soils will tend to be more robust to weather variability. They will drain well while retaining water in deeper layers in the event of drought or severe heat. Soil quality metrics that seek to directly measure physical, chemical and biological soil traits also emphasize buffering, storage and resilience. Examples include organic matter content, which acts as a sponge while promoting aeration and biological activity, and soil cationexchange capacity (CEC), which measures a soil's ability to bond essential nutrients over time for later use. Soil depth and organic matter content promote CEC (Ketterings, Reid and Rao 2007).

We posit an aggregate index of weather suitability for crop growth as variable $w$ with distribution $F(w)$ on support $\left[w_{l}, w_{h}\right]$ where we define $\bar{F}(w) \equiv 1-F(w)$. A land unit's ability to convert realized weather into yield is assumed to depend on the soil's quality. When $w=w_{h}$, then we assume that all land units yield quantity $y_{h}$. We parameterize soil quality as $\theta, \theta<w_{h}$, and yield response to weather conditions on $\left[\theta, w_{h}\right]$ is given as $\beta_{l}$. Here, a smaller value of $\theta$ indicates better land quality as the soils prove to be more resilient to adverse weather outcomes. However, yield is more sensitive to weather on interval $\left[w_{l}, \theta\right)$ and the response is given as $\beta_{h}$, $\beta_{h}>\beta_{l}$. Thus we posit the production relation:

$$
y(w ; \theta)= \begin{cases}\bar{y}-\beta_{l}\left(w_{h}-w\right), & w \in\left[\theta, w_{h}\right] \\ \bar{y}-\beta_{h}(\theta-w)-\beta_{l}\left(w_{h}-\theta\right), & w \in\left[w_{l}, \theta\right)\end{cases}
$$

where $\theta$ represents the point of non-differentiability. Figure 1 depicts. For convenience we assume that land quality and weather outcomes are never such that realized yield is zero. In the figure $\theta^{a}<\theta^{b}$ so that the better land, with $\theta=\theta^{a}$, has low sensitivity to adverse weather over the weather outcome set $\left[\theta^{a}, w_{h}\right]$, and a larger set than $\left[\theta^{b}, w_{h}\right]$. We refer to the model above as the land yield resilience model (LYRM) as its salient feature is that yield on better quality land is more resilient to bad weather. 


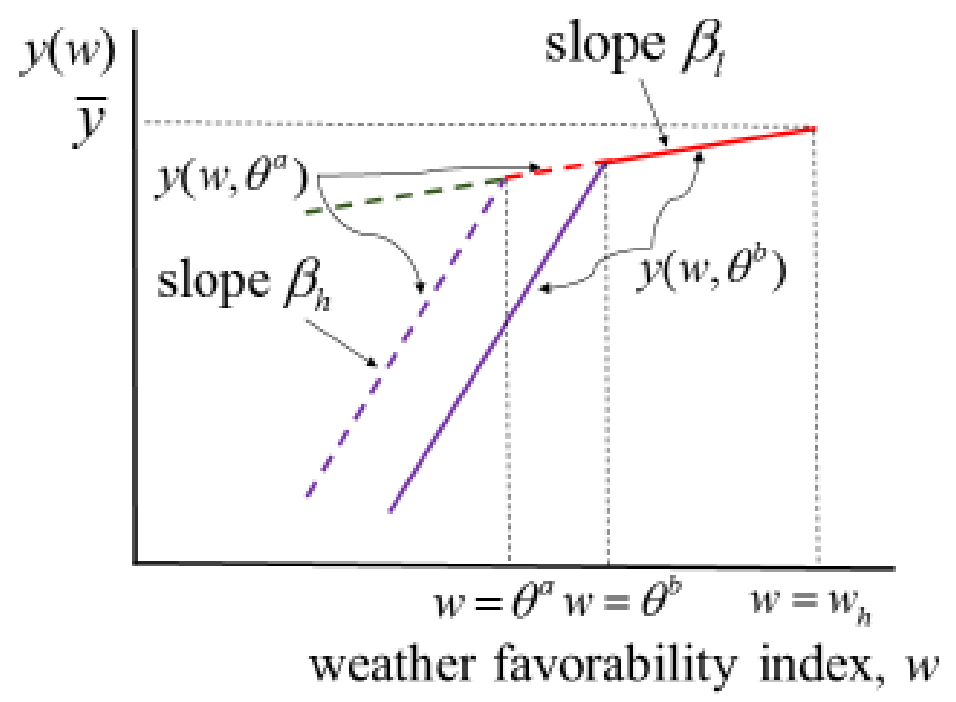

Figure 1. Yield, Weather Favorability, Soil Quality Relation

We note as a matter of model validation that mean yield is weakly decreasing in $\theta$, or

$d \int_{w_{l}}^{w_{h}} y(w ; \theta) d F(w) / d \theta \leq 0$. In addition, it is readily shown that the coefficient of yield variation will be larger under $\theta^{b}$ than under $\theta^{a}$. In that sense, the model is consistent with higher actuarial risk per bushel insured on poor quality land than on better quality land (Du et al. 2015).

The impact that higher $\theta$ (i.e., lower land quality) has on skewness is also apparent. Figure 2 shows how the weather favorability distribution maps into the yield distribution. The weather index cumulative distribution function is on the horizontal axis while that of the yield distribution has domain on the vertical axis (i.e., rotated counterclockwise by 90 degrees). The distributions have the same shape in the lower tail, up to location and scaling constants. In the upper tail, however, yield is not as sensitive to weather and so the yield distribution is more compact at its upper quantiles when compared with its lower quantiles than are the weather distribution's upper quantiles when compared with its lower quantiles. Comparative right-tail compaction is one way to characterize comparatively more negatively skewed distributions (Hao and Naiman 2007). 


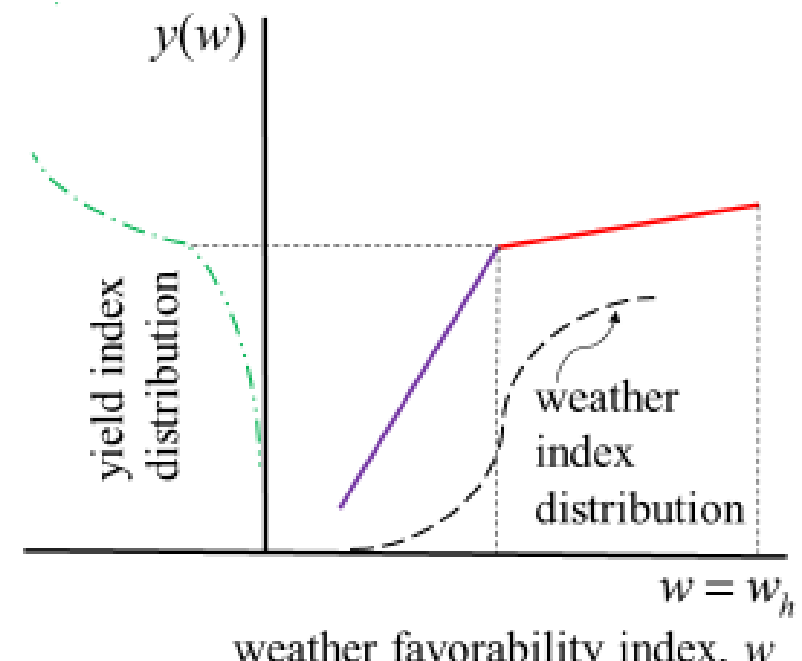

Figure 2. Map between Weather Index Distribution and Yield Distribution

Now, fixing on some weather distribution, we consider what effects an increase in land quality will have on the distribution's shape. There will be three effects. One is a rightward shift in the overall distribution (i.e., first-order stochastic dominance) as reflected by the fact that $y\left(w ; \theta^{a}\right) \geq y\left(w ; \theta^{b}\right)$ in Figure 1 . A second effect is lower variance, because $\beta_{l}$, rather than $\beta_{h}$, maps weather into yield over a larger $w$ set. There will also be further compaction of the right tail under $\theta^{a}$ when compared with $\theta^{b}$. This ensures that mean increases, variance decreases and, as the right tail is compacted, skewness becomes less positive or more negative. The latter point is consistent with findings in Du, Hennessy and Yu (2012) regarding how nitrogen (a substitute for land quality) affects yield skewness and in Du et al. (2015) regarding how land quality affects skewness.

Given that the model has only one source of randomness, weather favorability index $w$, one might ask whether yields are essentially perfectly correlated as a result. They are not, although they are comonotonic (i.e., they are monotone increasing functions of the same random variable) and so are perfectly ordinally correlated (p. 157 in Deelstra, Dhaene and Vanmaele 2011). One consequence is that $\lambda_{L} \equiv \lambda_{U} \equiv 1$ under the LYRM and so joint yield distributions 
cannot possibly be represented by the Gaussian copula. Another consequence is that the standard cardinal, or Pearson, correlation is not unity when units of land are of different quality. In Figure 1, when $\theta^{a}<w<\theta^{b}$, then yield on lower quality land is comparatively more sensitive to weather. As a result we have $\operatorname{corr}\left[y\left(w ; \theta^{a}\right), y\left(w ; \theta^{b}\right) \mid w \leq \theta^{b}\right] \leq \operatorname{corr}\left[y\left(w ; \theta^{a}\right), y\left(w ; \theta^{b}\right) \mid w \leq \theta^{a}\right] \equiv 1$ and $\operatorname{corr}\left[y\left(w ; \theta^{a}\right), y\left(w ; \theta^{b}\right) \mid \theta^{a} \leq w\right] \leq \operatorname{corr}\left[y\left(w ; \theta^{a}\right), y\left(w ; \theta^{b}\right) \mid \theta^{b} \leq w\right] \equiv 1$. More generally, the model supports the idea that correlations are weakest between the two tails.

Example 2: As an alternative to the piecewise linear setup in (5) we posit the set of production technologies given by

$$
y_{1}=\kappa_{0,1}-\kappa_{1,1} e^{-\lambda\left(w-\theta_{1}\right)} ; \quad y_{2}=\kappa_{0,2}-\kappa_{1,2} e^{-\lambda\left(w-\theta_{2}\right)} ;
$$

where $\kappa_{0,1}>0, \kappa_{0,2}>0, \kappa_{1,1}>0, \kappa_{1,2}>0$ and can have productivity interpretations while the $\theta_{i}$ continues to represent productivity. We will use this exponential function specification as a benchmark for comparison with a generalized version of (5). Yield correlation $\rho\left(y_{1}, y_{2}\right)=$ $\operatorname{cov}\left(y_{1}, y_{2}\right) /\left(\sqrt{\operatorname{var}\left(y_{1}\right)} \sqrt{\operatorname{var}\left(y_{2}\right)}\right)$ is given by

$$
\rho\left(y_{1}, y_{2}\right)=\frac{\mathbb{E}\left[e^{-\lambda\left(w-\theta_{1}\right)} e^{-\lambda\left(w-\theta_{2}\right)}\right]-\mathbb{E}\left[e^{-\lambda\left(w-\theta_{1}\right)}\right] \mathbb{E}\left[e^{-\lambda\left(w-\theta_{2}\right)}\right]}{\sqrt{\mathbb{E}\left[e^{-2 \lambda\left(w-\theta_{1}\right)}\right]-\left(\mathbb{E}\left[e^{-\lambda\left(w-\theta_{1}\right)}\right]\right)^{2}} \sqrt{\mathbb{E}\left[e^{-2 \lambda\left(w-\theta_{2}\right)}\right]-\left(\mathbb{E}\left[e^{-\lambda\left(w-\theta_{2}\right)}\right]\right)^{2}}}=1,
$$

where $\mathbb{E}[\cdot]$ is the expectation operator. The calculation tells us that there exists a class of production functions such that the common distribution of weather conditions, as represented by $F(w)$ with mean $\mu=\int w d F(w)$, and land heterogeneities, as represented by different values of $\theta$, do not affect yield correlations. In particular, correlation would remain perfect if one were to condition weather states toward either its lower or its upper tail and consider $\rho\left(y_{1}, y_{2} \mid w \leq \hat{w}\right)$ or $\rho\left(y_{1}, y_{2} \mid w \geq \hat{w}\right)$. Is this a general finding? We claim that it is not, because the calculation in (7) 
relies crucially on the constant and common absolute curvatures, $\lambda$, expressed in (6). Relative curvature is central to the determination of correlation.

\section{Generalized Land Yield Resilience and Likely Curvature}

Production functions are more likely to possess increasing relative curvature than constant or decreasing relative curvature. To see why, consider eq. (5) from above under the presence of random environmental noise $\varepsilon$, with $\mathbb{E}[\varepsilon]=0$;

$$
\begin{aligned}
Y(w, \varepsilon ; \theta) & = \begin{cases}\bar{Y}-\beta_{l}\left(w_{h}-w-\varepsilon\right), & w \in\left[\theta-\varepsilon, w_{h}\right] ; \\
\bar{Y}-\beta_{h}(\theta-w-\varepsilon)-\beta_{l}\left(w_{h}-\theta\right), & w \in\left[w_{l}, \theta-\varepsilon\right) ;\end{cases} \\
& =\bar{Y}-\beta_{l}\left(w_{h}-w-\varepsilon\right)-\left(\beta_{h}-\beta_{l}\right) \max [\theta-w-\varepsilon, 0] .
\end{aligned}
$$

Figure 1 depicts for a fixed value of $\varepsilon$. However, as in Berck and Helfand (1990), we will integrate out over $\varepsilon$ in order to smooth out the point of non-differentiability at $w=\theta$ where we let $\varepsilon \sim K(\varepsilon)$ with density $k(\varepsilon) .{ }^{3}$ Thus, and using an integration by parts,

$$
y_{\theta}(w) \equiv \int Y(w, \varepsilon ; \theta) d K(\varepsilon)=\bar{Y}-\beta_{l}\left(w_{h}-w\right)+\left(\beta_{h}-\beta_{l}\right) \int_{-\infty}^{\theta-w} K(\varepsilon) d \varepsilon
$$

We refer to production function (9) as our generalized land yield resilience model (GRM).

The curvature of $y_{\theta}(w)$ in $w$ is given by

$$
-\frac{y_{\theta}^{\prime \prime}}{y_{\theta}^{\prime}}=\frac{k(\theta-w)}{K(\theta-w)}=\frac{d \ln [K(\theta-w)]}{d \theta},
$$

where primes are used to denote order of differentiation. Now

$$
\frac{d\left(-y_{\theta}^{\prime \prime} / y_{\theta}^{\prime}\right)}{d w}=-\frac{d^{2} \ln [K(\theta-w)]}{d \theta^{2}}=-\frac{\left[K(\theta-w) k^{\prime}(\theta-w)-[k(\theta-w)]^{2}\right]}{[K(\theta-w)]^{2}} .
$$

As has been pointed out in Du et al. (2015), if some $K(z)$ is a distribution function and

\footnotetext{
${ }^{3}$ In line with the Berck and Helfand (1990), the $\varepsilon$ may be interpreted as local heterogeneities in land quality, as true local randomness in weather or an endeavor to generalize the production relationship. For our purposes the distinctions are not relevant.
} 
$k(z) / K(z)$ is increasing over its support, then density $k(z)$ must be increasing over its support. Thus, $d\left(-y_{\theta}^{\prime \prime} / y_{\theta}^{\prime}\right) / d w \leq 0$ (i.e., decreasing curvature) on the support is very unlikely. However, $d\left(-y_{\theta}^{\prime \prime} / y_{\theta}^{\prime}\right) / d w \geq 0$ (i.e., logconcavity of $\left.K(\cdot)\right)$ or $d^{2} \ln [K(\theta-w)] / d \theta^{2} \leq 0$, on $w \in\left(w_{l}, w_{h}\right)$ is much more plausible as smoothing functions tend to taper weightings at the tail in a manner consistent with logconcavity. Most, if not all, common smoothing kernels satisfy $d^{2} \ln [K(\theta-w)] / d \theta^{2} \leq 0$. See Bagnoli and Bergstrom (2005) for more details on logconcavity.

\section{Second-order Analysis and Unconditional Correlation}

We turn now to a second-order Taylor series expansion of a twice smoothly differentiable, but otherwise arbitrary, yield function of form $y_{\theta}(w)$ around mean $\mu$, where higher $\theta$ represents lower land productivity. By 'arbitrary’ we mean that it need not be GYRM in the manner of (9). The expansion gives

$$
\begin{aligned}
& y_{\theta}(w) \approx \bar{y}_{\theta}+\tilde{w} \bar{y}_{\theta}^{\prime}+0.5 \tilde{w}^{2} \bar{y}_{\theta}^{\prime \prime} ; \quad \tilde{w} \equiv w-\mu ; \\
& \left.\bar{y}_{\theta} \equiv y_{\theta}(w)\right|_{w=\mu} ;\left.\quad \bar{y}_{\theta}^{\prime} \equiv y_{\theta}^{\prime}(w)\right|_{w=\mu} ;\left.\quad \bar{y}_{\theta}^{\prime \prime} \equiv y_{\theta}^{\prime \prime}(w)\right|_{w=\mu} .
\end{aligned}
$$

Let $\sigma^{2}=\mathbb{E}\left[\tilde{w}^{2}\right]$ be variance, let $\alpha=\mathbb{E}\left[\tilde{w}^{3}\right]$ be the non-normalized third central moment (and so, respectively, negative or positive when the weather index distribution is negatively or positively skewed), and let $\beta=\mathbb{E}\left[\tilde{w}^{4}\right]-\sigma^{4}$ represent (non-normalized) kurtosis. ${ }^{4}$ Thus,

\footnotetext{
${ }^{4}$ Pearson skewness is given by $\alpha / \sigma^{3 / 2}=\mathbb{E}\left[\tilde{w}^{3}\right] / \sigma^{3}$ while Pearson kurtosis is given by $\mathbb{E}\left[\tilde{w}^{4}\right] / \sigma^{4}$. Pearson excess kurtosis is given by $\left(\mathbb{E}\left[\tilde{w}^{4}\right]-3 \sigma^{4}\right) / \sigma^{4}$. The normal distribution has 3 as kurtosis statistic so that excess kurtosis measures kurtosis relative to the normal distribution. It is known that $\mathbb{E}\left[\tilde{w}^{4}\right] / \sigma^{4} \geq 1$ with equality only for the equi-probable two-point distribution. Also, centralized moments must adhere to the inequality $\left\{\mathbb{E}\left[\tilde{w}^{4}\right] / \sigma^{4}\right\} \geq\left(\mathbb{E}\left[\tilde{w}^{3}\right] / \sigma^{3}\right)^{2}+1$.
} 


$$
\begin{aligned}
& \operatorname{cov}\left(y_{\theta_{1}}, y_{\theta_{2}}\right) \approx \operatorname{cov}\left[\tilde{w} \bar{y}_{\theta_{1}}^{\prime}+0.5 \tilde{w}^{2} \bar{y}_{\theta_{1}}^{\prime \prime}, \tilde{w} \bar{y}_{\theta_{2}}^{\prime}+0.5 \tilde{w}^{2} \bar{y}_{\theta_{2}}^{\prime \prime}\right] \\
& =\bar{y}_{\theta_{1}}^{\prime} \bar{y}_{\theta_{2}}^{\prime} \sigma^{2}+0.5 \alpha\left[\bar{y}_{\theta_{1}}^{\prime} \bar{y}_{\theta_{2}}^{\prime \prime}+\bar{y}_{\theta_{1}}^{\prime \prime} \bar{y}_{\theta_{2}}^{\prime}\right]+0.25 \beta \bar{y}_{\theta_{1}}^{\prime \prime} \bar{y}_{\theta_{2}}^{\prime \prime} ; \\
& \operatorname{var}\left(y_{\theta_{i}}\right) \approx \operatorname{var}\left[\tilde{w} \bar{y}_{\theta_{i}}^{\prime}+0.5 \tilde{w}^{2} \bar{y}_{\theta_{i}}^{\prime \prime}\right]=\left[\bar{y}_{\theta_{i}}^{\prime}\right]^{2} \sigma^{2}+\alpha \bar{y}_{\theta_{i}}^{\prime} \bar{y}_{\theta_{i}}^{\prime \prime}+0.25 \beta\left[\bar{y}_{\theta_{i}}^{\prime \prime}\right]^{2}, \quad i \in\{1,2\} .
\end{aligned}
$$

Let $\lambda_{i}=-\bar{y}_{\theta_{i}}^{\prime \prime} / \bar{y}_{\theta_{i}}^{\prime}>0$ represent the relative curvature function for land of quality $\theta_{i}$ so that correlation between land units of types $\theta_{1}$ and $\theta_{2}$ is, to an approximation,

$$
\rho_{\theta_{1}, \theta_{2}} \approx \hat{\rho}_{\theta_{1}, \theta_{2}} \equiv \frac{4 \sigma^{2}-2 \alpha\left[\lambda_{1}+\lambda_{2}\right]+\beta \lambda_{1} \lambda_{2}}{\sqrt{4 \sigma^{2}-4 \alpha \lambda_{1}+\beta \lambda_{1}^{2}} \sqrt{4 \sigma^{2}-4 \alpha \lambda_{2}+\beta \lambda_{2}^{2}}} .
$$

Now consider the following cases:

i) constant absolute curvature production functions with $\lambda_{1}=\lambda_{2}$. Then, regardless of the crop condition distribution, $\rho_{\theta_{1}, \theta_{2}}=1$ as in (6) above.

ii) weather index symmetry, in which instance skewness is zero and $\alpha=0$. Then

$$
\hat{\rho}_{\theta_{1}, \theta_{2}}=\frac{4 \sigma^{2}+\beta \lambda_{1} \lambda_{2}}{\sqrt{4 \sigma^{2}+\beta \lambda_{1}^{2}} \sqrt{4 \sigma^{2}+\beta \lambda_{2}^{2}}} \equiv \begin{cases}1 & \text { iff } \lambda_{1}=\lambda_{2} ; \\ <1 & \text { otherwise }\end{cases}
$$

With $\alpha=0$, then

$$
\frac{d \hat{\rho}_{\theta_{1}, \theta_{2}}}{d \lambda_{1}}=\frac{4 \beta \sigma^{2}\left(\lambda_{2}-\lambda_{1}\right)}{\left(4 \sigma^{2}+\beta \lambda_{1}^{2}\right)^{3 / 2} \sqrt{4 \sigma^{2}+\beta \lambda_{2}^{2}}} \stackrel{\text { sign }}{=} \lambda_{2}-\lambda_{1} .
$$

An increase in one function's relative curvature decreases the correlation estimate if and only if that relative curvature is the larger of the two. This comparative static is consistent with the notion that the unconditional correlation between yields decreases whenever the two yield functions become more dissimilar.

iii) where $\lambda_{1} \rightarrow \infty$ but $\lambda_{2} \in(0, \infty)$. Then both numerator and denominator in (14) have limiting value of $\infty$ so we apply L'Hospital's rule (p. 109 in Rudin 1976):

$$
\left.\hat{\rho}_{\theta_{1}, \theta_{2}} \rightarrow \frac{\left(\beta \lambda_{2}-2 \alpha\right) \sqrt{4 \sigma^{2}-4 \alpha \lambda_{1}+\beta \lambda_{1}^{2}}}{\left(\beta \lambda_{1}-2 \alpha\right) \sqrt{4 \sigma^{2}-4 \alpha \lambda_{2}+\beta \lambda_{2}^{2}}}\right|_{\lambda_{1} \rightarrow \infty}=\frac{1}{\sqrt{1+4 \frac{\left(\beta \sigma^{2}-\alpha^{2}\right)}{\left(\beta \lambda_{2}-2 \alpha\right)^{2}}}} .
$$


Now we know from $\left\{\mathbb{E}\left[\tilde{w}^{4}\right] / \sigma^{4}\right\} \geq\left(\mathbb{E}\left[\tilde{w}^{3}\right] / \sigma^{3}\right)^{2}+1$ in footnote 4 that $\beta \sigma^{2} \geq \alpha^{2}$ so that we may view $\left(\beta \sigma^{2}-\alpha^{2}\right) /\left(\beta \lambda_{2}-2 \alpha\right)^{2}$ as an index of uncorrelatedness under $\lambda_{2} \in(0, \infty)$ and $\lambda_{1} \rightarrow \infty$. The estimate of correlation declines when variance increases, because an increase in weather variance matters greatly for yield under the function with low curvature, but less so for yield under the other function. For $\lambda_{2}$ sufficiently high, namely $\beta \lambda_{2}>2 \alpha$, compare too when the value of $\alpha$ changes from $\alpha=\hat{\alpha}>0$ to $\alpha=-\hat{\alpha}<0$. Then the correlation estimate in (17) increases. When compared with positive skewness, negative skewness offsets the impact of production function heterogeneity on the yield correlation estimate. In light of this observation alone, to the extent that poorer growing regions have more positively skewed yields, as is quite well-documented in the literature (Hennessy 2009; Boyer, Brorsen and Tumusiime 2015), we should see stronger unconditional correlations among yields in the better growing region.

iv) where $\lambda_{2} \rightarrow 0$ but $\lambda_{1} \in(0, \infty)$. Then

$$
\hat{\rho}_{\theta_{1}, \theta_{2}} \rightarrow \frac{2 \sigma^{2}-\alpha \lambda_{1}}{\sigma \sqrt{4 \sigma^{2}-4 \alpha \lambda_{1}+\beta \lambda_{1}^{2}}}=\frac{1}{\sqrt{1+\frac{\left(\beta \sigma^{2}-\alpha^{2}\right)}{\left(\frac{2 \sigma^{2}}{\lambda_{1}}-\alpha\right)^{2}}}}
$$

Although the effect of an increase in variance is unclear in this situation, if the $\alpha$ value changes from $\alpha=\hat{\alpha}>0$ to $\alpha=-\widehat{\alpha}<0$ then the correlation estimate in (18) increases, as had been the case with (17). Note too that if $\lambda_{1} \rightarrow \infty$, then $\hat{\rho}_{\theta_{1}, \theta_{2}} \rightarrow 1 / \sqrt{\beta \sigma^{2} / \alpha^{2}} \equiv|\alpha| /\left(\sigma \beta^{0.5}\right)$, so that the absolute magnitude of skewness (and not the sign) determines correlation. Even though the production functions have very different curvatures, most of what determines correlation is out in one or other tail of the weather index distribution and so unconditional correlation will be high when the distribution is strongly skewed.

$v$ ) where $\lambda_{1}=\lambda, \lambda_{2}=\tau \lambda$ and $\lambda \rightarrow \infty$. In this situation land is still heterogeneous but, according to the GRM, land is generally of lower quality. Then 


$$
\begin{aligned}
& \hat{\rho}_{\theta_{1}, \theta_{2}}=\frac{4 \sigma^{2}-2 \alpha[\lambda+\tau \lambda]+\beta \tau \lambda^{2}}{\sqrt{4 \sigma^{2}-4 \alpha \lambda+\beta \lambda^{2}} \sqrt{4 \sigma^{2}-4 \alpha \tau \lambda+\beta \tau^{2} \lambda^{2}}} \\
& \left.\rightarrow \frac{\frac{4 \sigma^{2}}{\lambda^{2}}-\frac{2 \alpha(1+\tau)}{\lambda}+\beta \tau}{\sqrt{\frac{4 \sigma^{2}}{\lambda^{2}}-\frac{\alpha}{\lambda}+\beta \sqrt{\frac{4 \sigma^{2}}{\lambda^{2}}-\frac{4 \alpha \tau}{\lambda}+\beta \tau^{2}}}}\right|_{\lambda \rightarrow \infty}=\frac{\beta \tau}{\sqrt{\beta} \sqrt{\beta \tau^{2}}}=1 .
\end{aligned}
$$

Thus, in poor land regions one would expect stronger yield correlations at the limit. This is as to be expected, because higher yield responsiveness to weather favorability dominates for both plots of land.

vi) where $\lambda_{1}=\lambda, \lambda_{2}=\tau \lambda$ and $\lambda \rightarrow 0$ so that land is still heterogeneous, but, according to the $d\left(-y_{\theta}^{\prime \prime} / y_{\theta}^{\prime}\right) / d w \geq 0$ property in the GRM, land is generally of higher quality. Then

$$
\hat{\rho}_{\theta_{1}, \theta_{2}}=\frac{4 \sigma^{2}-2 \alpha[\lambda+\tau \lambda]+\beta \tau \lambda^{2}}{\sqrt{4 \sigma^{2}-4 \alpha \lambda+\beta \lambda^{2}} \sqrt{4 \sigma^{2}-4 \alpha \tau \lambda+\beta \tau^{2} \lambda^{2}}} \rightarrow 1
$$

This also is to be expected, because lower yield responsiveness to weather favorability dominates for both plots of land.

When viewed together, cases $v$ ) and vi) show that correlation should be U shaped. It should be high when land is heterogeneous but on the whole of low quality and also when it is heterogeneous but on the whole of high quality. Cases ii), iii) and iv) hold that correlation should not be as high when land is of intermediate quality.

\section{Second-order Analysis and Conditional Correlation}

The above analysis asks about unconditional correlation, but our primary interest regards correlation in the left tails. To further our inquiries, suppose now that cumulative weather favorability emerges over the season as a martingale (Durrett 1996) of the form

$$
w=\eta+\sum_{n=1}^{N} \omega_{n}
$$

where $\eta$ represents a land-specific soil endowment and one might view the $\omega_{n}$ to be growing 
degrees per period over an $N$ period season. Without loss of generality we can (and will) assume that $\mathbb{E}\left[\omega_{n}\right]=0$, while we also impose i.i.d. structure on the $\omega_{n}$, so that we may (and will) represent any one $\omega_{n}$ by $\omega$.

Define residual variability over the growing season by $w_{m}=\sum_{n=m}^{N} \omega_{n}$ with conditional centralized moments $\alpha_{m}=\mathbb{E}\left[w_{m}^{3}\right]$ and $\beta_{m}=\mathbb{E}\left[w_{m}^{4}\right]-\left(\mathbb{E}\left[w_{m}^{2}\right]-\left(\mathbb{E}\left[w_{m}\right]\right)^{2}\right)^{2}$. Specifying $R \equiv$ $N-m+1$, calculations in Appendix A show the intra-season period analog of (14) to be

$$
\hat{\rho}_{m, \theta_{1}, \theta_{2}}=\frac{4 \sigma^{2}-2 \mathbb{E}\left[\omega^{3}\right]\left[\lambda_{1}+\lambda_{2}\right]+\mathbb{E}\left[\omega^{4}\right] \lambda_{1} \lambda_{2}+5 R \sigma^{4} \lambda_{1} \lambda_{2}}{\sqrt{4 \sigma^{2}-4 \mathbb{E}\left[\omega^{3}\right] \lambda_{1}+\left(\mathbb{E}\left[\omega^{4}\right]+5 R \sigma^{4}\right) \lambda_{1}^{2}} \sqrt{4 \sigma^{2}-4 \mathbb{E}\left[\omega^{3}\right] \lambda_{2}+\left(\mathbb{E}\left[\omega^{4}\right]+5 R \sigma^{4}\right) \lambda_{2}^{2}}} .
$$

Were $N+1$ much larger than $m$, such that $R$ is large, which might be interpreted as early in the season, then many of the terms will have smaller values and $\hat{\rho}_{m, \theta_{1}, \theta_{2}}$ will be larger. Specifically, $\lim _{R \rightarrow \infty} \hat{\rho}_{m, \theta_{1}, \theta_{2}}=5 \sigma^{4} \lambda_{1} \lambda_{2} /\left(\sqrt{5 \sigma^{4} \lambda_{1}^{2}} \sqrt{5 \sigma^{4} \lambda_{2}^{2}}\right)=1$. The intuition here is that higher levels of uncertainty, as in early season, make it unclear as to whether the marginal response will be $\beta_{h}$ or $\beta_{l}$. However, later in the season, in the presence of more information, the likelihood that $w$ will be such that both land types suffer severe yield losses, both escape severe weather losses, or lower quality land only suffers severe weather losses becomes more certain and the range for conditional correlations becomes more dispersed.

Let us now consider each possible outcome in turn. Suppose that the accumulation of soil endowment plus early growing season shocks, $\eta+\sum_{n=1}^{m-1} \omega_{n}$, is large (good land, good early weather or good conditions going into planting) so that we are considering the right tail correlation. If GRM applies then both $\lambda_{1}$ and $\lambda_{2}$ are large and Case $v$ ) above suggests that the right tail correlation should be high. One might view this as a good or normal outcome for a prime growing region such as Iowa. Alternatively, suppose that $\eta+\sum_{n=1}^{m-1} \omega_{n}$ is small so that the 
left tail correlation is being considered. If GRM applies then both $\lambda_{1}$ and $\lambda_{2}$ are small and Case vi) suggests that the correlation should also be high. One might view this as a poor outcome for more marginal land, as in the Western Great Plains, or perhaps a very poor outcome in locations with better land. Finally, suppose that $\eta+\sum_{n=1}^{m-1} \omega_{n}$ is intermediate so that the context is a moderate or poor outcome in good land areas or a good outcome in poor land areas. Then the conditional correlation should be lower because a) low-end land in good areas will see yield failures but moderate and better quality land will hold up, whereas b) low-end and moderate quality land in poor land areas will still fail, while good quality land in these areas will not see yield failures. Thus, the model is consistent with our earlier graphical interpretation.

\section{Tail Dependence}

Consider now our GRM above, where we let $x_{1} \equiv y\left(w ; \theta^{a}\right)$ and $x_{2} \equiv y\left(w ; \theta^{b}\right)$. We seek to show that Condition (1) applies, in other words,

$$
P\left[y\left(w ; \theta^{b}\right) \leq \hat{x}_{2} \mid y\left(w ; \theta^{a}\right) \leq \hat{x}_{1}\right] \text { is nonincreasing in } \hat{x}_{1} \text {, for all } \hat{x}_{2} .
$$

Figure 3 depicts where $\hat{x}_{2}$ is arbitrarily chosen and $\hat{w}_{2}$ is the corresponding weather index value for $y\left(w ; \theta^{b}\right)$ so that $P\left[y\left(w ; \theta^{b}\right) \leq \hat{x}_{2}\right]=F\left(\hat{w}_{2}\right)$. When $\hat{x}_{1}=\hat{x}_{1}^{*}$ with corresponding weather quantile $\hat{w}_{1}^{*}$, then $P\left[y\left(w ; \theta^{a}\right) \leq \hat{x}_{1}^{*}\right]=P\left[w \leq \hat{w}_{1}^{*}\right]=F\left(\hat{w}_{1}^{*}\right)$. In this case, whenever weather conditions are sufficiently bad to ensure that $y\left(w ; \theta^{a}\right) \leq \hat{x}_{1}^{*}$, then they will be sufficiently bad to ensure that $y\left(w ; \theta^{b}\right) \leq \hat{x}_{2}$ and so $P\left[y\left(w ; \theta^{b}\right) \leq \hat{x}_{2} \mid y\left(w ; \theta^{a}\right) \leq \hat{x}_{1}^{*}\right]=1$. However, when $\hat{x}_{1}=\hat{x}_{1}^{* *}$, then $y\left(w ; \theta^{b}\right) \leq \hat{x}_{2}$ is no longer guaranteed. More generally,

$$
\begin{aligned}
& P\left[y\left(w ; \theta^{b}\right) \leq \hat{x}_{2} \mid y\left(w ; \theta^{a}\right) \leq \hat{x}_{1}\right]=\frac{P\left[\left\{y\left(w ; \theta^{b}\right) \leq \hat{x}_{2}\right\} \cap\left\{y\left(w ; \theta^{a}\right) \leq \hat{x}_{1}\right\}\right]}{P\left[y\left(w ; \theta^{a}\right) \leq \hat{x}_{1}\right]} \\
& =\frac{\min \left[F\left(\hat{w}_{2}\right), F\left(\hat{w}_{1}\right)\right]}{F\left(\hat{w}_{1}\right)}=\min \left[\frac{F\left(\hat{w}_{2}\right)}{F\left(\hat{w}_{1}\right)}, 1\right] .
\end{aligned}
$$




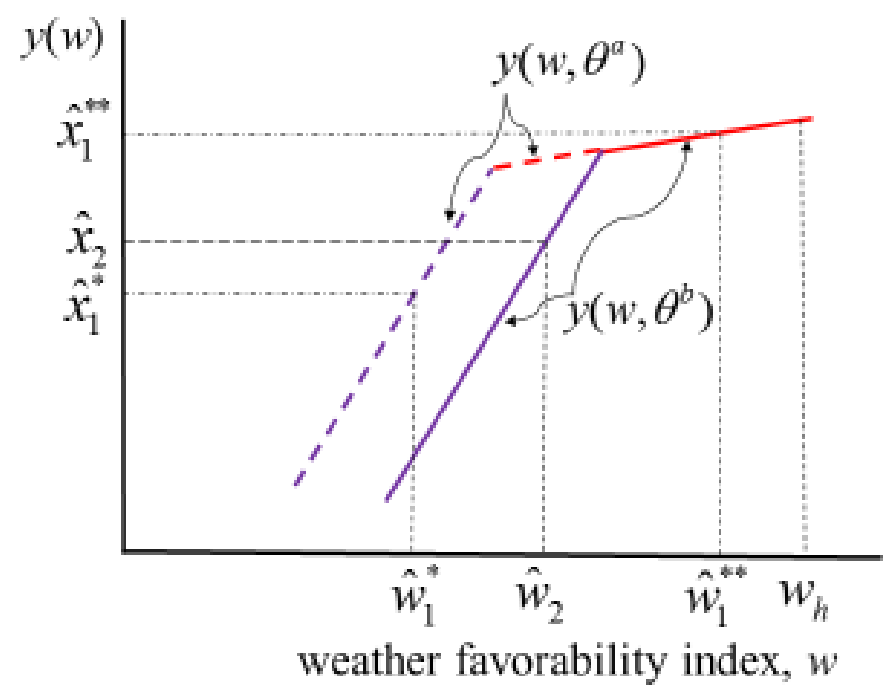

\section{Figure 3. Graphic of LTD Property for Land Yield Resilience Model}

Now $\hat{x}_{1}$ is monotone increasing in $\hat{w}_{1}$ and $F\left(\hat{w}_{2}\right) / F\left(\hat{w}_{1}\right)$ is nonincreasing in $\hat{w}_{1}$, so that $P\left[y\left(w ; \theta^{b}\right) \leq \hat{x}_{2} \mid y\left(w ; \theta^{a}\right) \leq \hat{x}_{1}\right]$ is nonincreasing in $\hat{x}_{1}$. Notice that the inference above is symmetric in that it continues to apply if we interchange yield positions and consider instead $P\left[y\left(w ; \theta^{a}\right) \leq \hat{x}_{2} \mid y\left(w ; \theta^{b}\right) \leq \hat{x}_{1}\right]$. We have shown:

Proposition 1: In the LYRM, all pairs of land units chosen have joint yield distributions that satisfy the LTD property.

Figure 4 describes the function $P\left[y\left(w ; \theta^{b}\right) \leq \hat{x}_{2} \mid y\left(w ; \theta^{a}\right) \leq \hat{x}_{1}\right]$ as $\hat{x}_{1}$ changes. We turn now to condition (2). Here we seek to show that

$$
P\left[y\left(w ; \theta^{b}\right) \geq \hat{x}_{2} \mid y\left(w ; \theta^{a}\right) \geq \hat{x}_{1}\right] \text { is nondecreasing in } \hat{x}_{1} \text {, for all } \hat{x}_{2} \text {. }
$$

The reasoning leading to (24) above now supports

$$
P\left[y\left(w ; \theta^{b}\right) \geq \hat{x}_{2} \mid y\left(w ; \theta^{a}\right) \geq \hat{x}_{1}\right]=\min \left[\frac{\bar{F}\left(\hat{w}_{2}\right)}{\bar{F}\left(\hat{w}_{1}\right)}, 1\right] .
$$




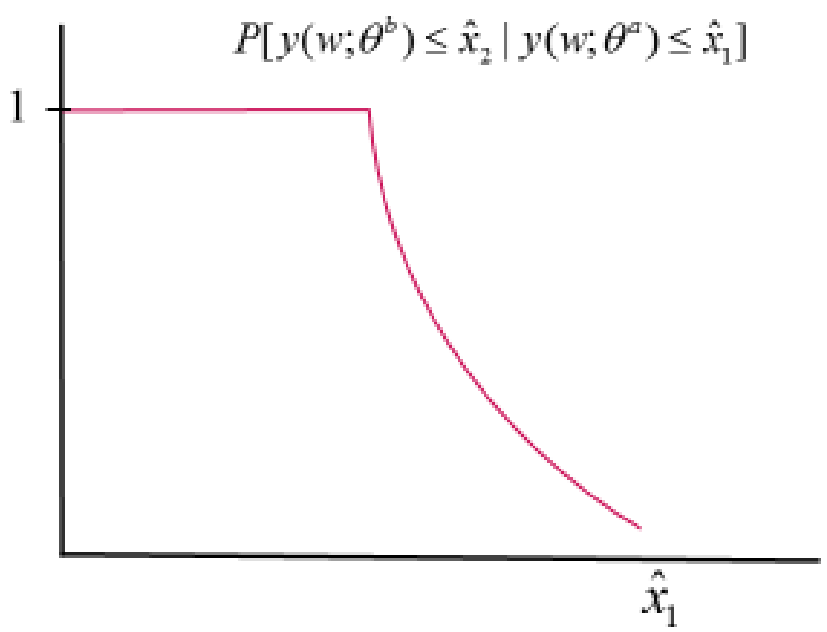

Figure 4. LTD function as the Conditioning Yield Changes

As $\hat{x}_{1}$ is monotone increasing in $\hat{w}_{1}$ and $\bar{F}\left(\hat{w}_{2}\right) / \bar{F}\left(\hat{w}_{1}\right)$ is nondecreasing in $\hat{w}_{1}$, we can conclude that $P\left[y\left(w ; \theta^{b}\right) \geq \hat{x}_{2} \mid y\left(w ; \theta^{a}\right) \geq \hat{x}_{1}\right]$ is nondecreasing in $\hat{x}_{1} \cdot{ }^{5}$

Proposition 2: In the LYRM, all pairs of land units chosen have joint yield distributions that satisfy the RTI property.

Finally, we adapt Figure 1 to consider how tail dependence can differ across states. The impact could be modeled in two ways. We could shift the weather favorability distribution to the left to indicate a less favorable weather environment or we could shift the yield functions to the right to indicate poorer soils. Figure 5 illustrates by shifting the weather favorability distribution where the left-most distribution is intended to illustrate South Dakota and the rightmost distribution is intended to illustrate Iowa, both for corn. In fact, both weather conditions and soils are generally better for corn in Iowa so we can, for ease of presentation and without compromise to representation, just assume that the distinction between the states is represented by a shift in the weather favorability distribution. In Iowa, the distribution is massed largely

5 These propositions are demonstrated in an intuitive way for the LYRM, but the arguments also apply to the GRM. 


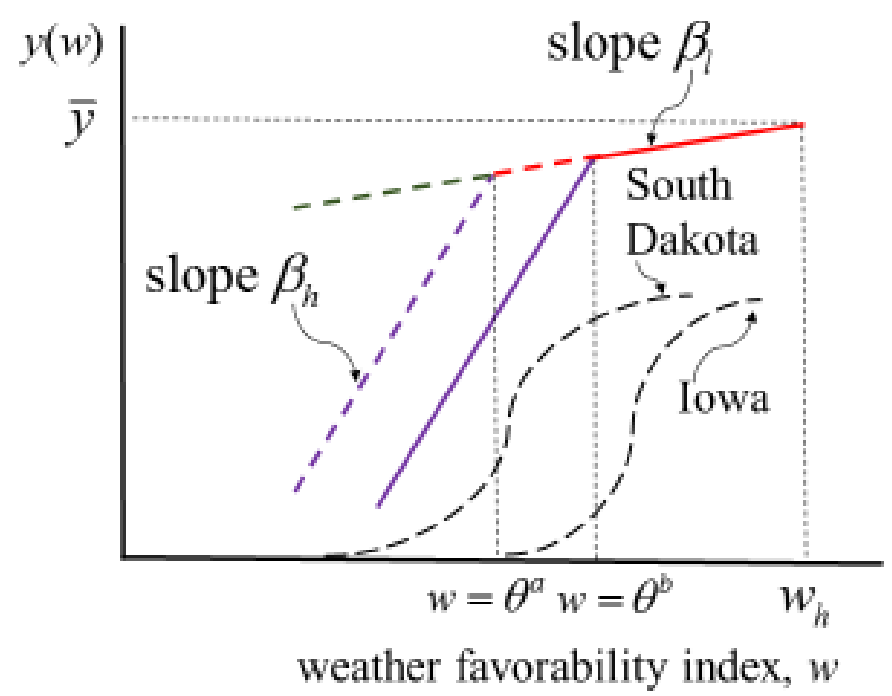

Figure 5. Characterizing Yield and Weather Favorability Differences across States

above the kinks and so reflects good general growing conditions and/or soils. As a result, with $w_{q}$ as the $q$ th quantile for weather, we would expect that conditional correlations follow

$$
\begin{aligned}
& \text { IA: }\left\{\frac{d \operatorname{corr}\left[y\left(w ; \theta^{b}\right), y\left(w ; \theta^{a}\right) \mid w \leq w_{q}\right]}{d w_{q}}>0, \quad \frac{d \operatorname{corr}\left[y\left(w ; \theta^{b}\right), y\left(w ; \theta^{a}\right) \mid w>w_{q}\right]}{d w_{q}}>0\right. \text {; } \\
& \mathrm{SD}:\left\{\frac{d \operatorname{corr}\left[y\left(w ; \theta^{b}\right), y\left(w ; \theta^{a}\right) \mid w \leq w_{q}\right]}{d w_{q}}<0, \quad \frac{d \operatorname{corr}\left[y\left(w ; \theta^{b}\right), y\left(w ; \theta^{a}\right) \mid w>w_{q}\right]}{d w_{q}}<0,\right.
\end{aligned}
$$

Empirical tests for these and other properties are laid out in the next section.

\section{Data}

Insurance unit-level corn yield data in 13 states (IL, IN, IA, KS, MI, MN, MO, NE, ND, OH, OK, SD, WI) over the period 1990-2009 are employed for this study. The data are obtained from the RMA. Each sample unit was insured under federal crop insurance programs over the sample period and has a yield record of up to 10 years, which can be non-consecutive for some units. For our purposes, to obtain the longest time series for yields we only select units with 10 year yield histories. In the 937 sampled counties, we have a total of 418,983 insurance units with average size and yield equaling 98 acres and 140.7 bushels per unit, respectively. 
Yields were observed over the period 1989-2008 and the majority of yield records pertain to 1993 or later.

As is commonly applied in the literature, detrending is intended to allow for yield outcome comparisons across years and is the first step in crop yield modelling. The objective is to control for time trend effects so that stochastic attributes of yields in different years can be compared. We use the multiplicative model in Claassen and Just (2011). The procedure is described briefly as follows:

(i) Use a non-parametric local regression to estimate county yield trend, denoted by $\hat{y}^{c}$. The tri-cube weighting kernel is used.

(ii) Estimate unit level trend yield, $\hat{y}^{u}$, in the multiplicative model, $\hat{y}^{u}=\phi^{u} \hat{y}^{c}$, where $\phi^{u}$ is the unit specific productivity measure.

(iii) Calculate unit level detrended yield from the observed yield $y$ as $\varepsilon^{u}=y-\hat{y}^{u}$.

Before formal statistical testing, we provide some visual representations of how yearmatched yield data correlate. The left-hand scatterplot in Figure 6 displays yields of a random
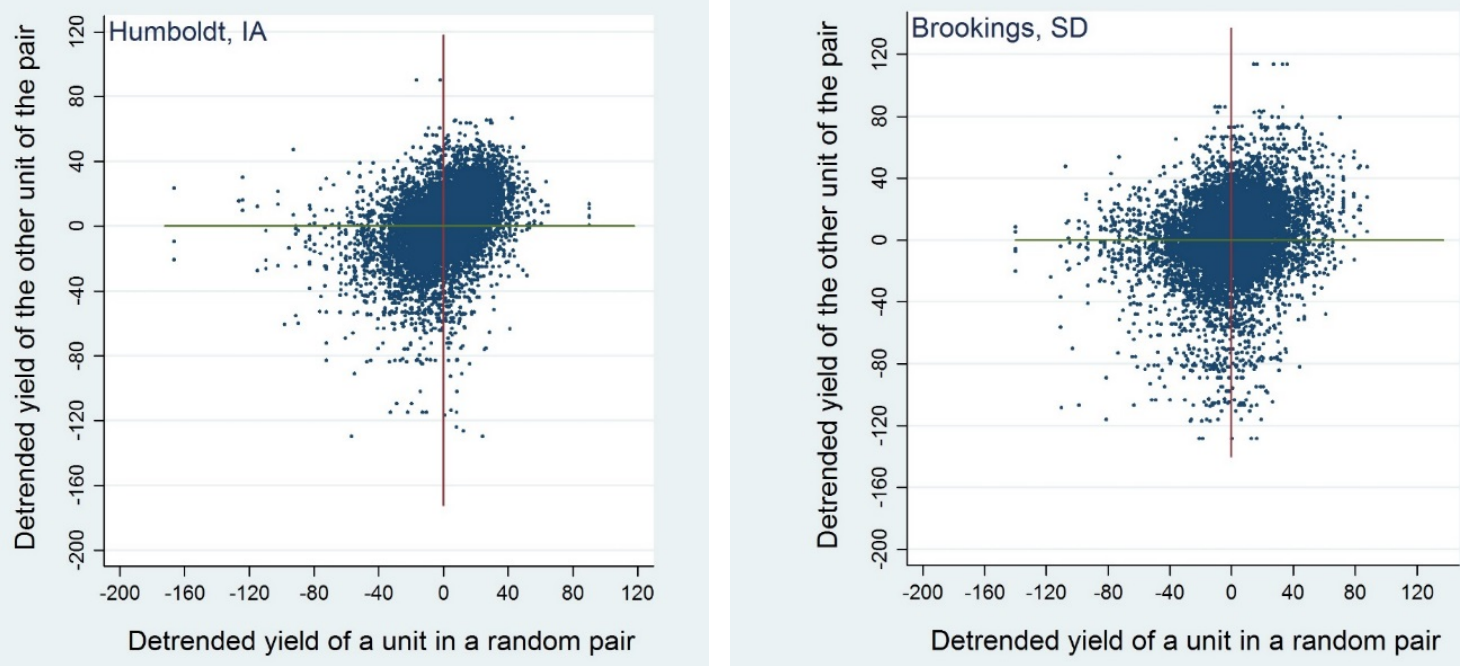

Note: The samples are 1,000 random pairs of year-matched units within each county.

Figure 6. For a County in Iowa and a County in South Dakota, Scatter Plot of Detrended Yields when Units within County are Randomly Matched 
sample of 1,000 year-matched yield pairs from Humboldt County in north-central Iowa, a county with good soils and growing conditions. The right-hand scatterplot provides the same data for Brookings County in east-central South Dakota, a county at the edge of the Corn Belt where corn production has challenged growers. In regard to corn production, the counties are quite typical of their region.

Several observations can be made based on the plots;

(i) yields are positively correlated across units.

(ii) yields are more dispersed in the third quadrant than in the first quadrant (i.e., yields vary more conditional on being below normal yield when compared with being above normal).

(iii) differences among plots suggest that there is regional heterogeneity in yield dependence, where Humboldt Co., Iowa, plots tend to be more tightly distributed around the $y_{2}=y_{1}$ bisector.

(iv) unlike the general presumption in the literature (Goodwin and Hungerford 2015), it is not apparent just from the figure that the correlation gets stronger at either the left tail or the right tail. In other words, there is no obvious evidence of tail dependence, especially at the left tail.

Point (iv) surprised the authors. Weather variation is the dominant cause of yield variation. Weather affects not just individual farms but all farms in a region, and so farm yields tend to vary systematically. One might infer then that extremely bad weather tends to cause extremely low yields across all units in affected regions. Thus, the authors expected to observe clear dependence at the left tail. On the other hand, because idiosyncratic risks tend to cancel each other out, we expect stronger dependence among county average yields. Figure 7 is the same as Figure 6 except that observations are randomly assembled into 50-unit groups and yields are averaged before randomly matching with yield averages from other 50-unit groups. A comparison of corresponding panels in Figures 6 and 7 shows that correlation is stronger for the 

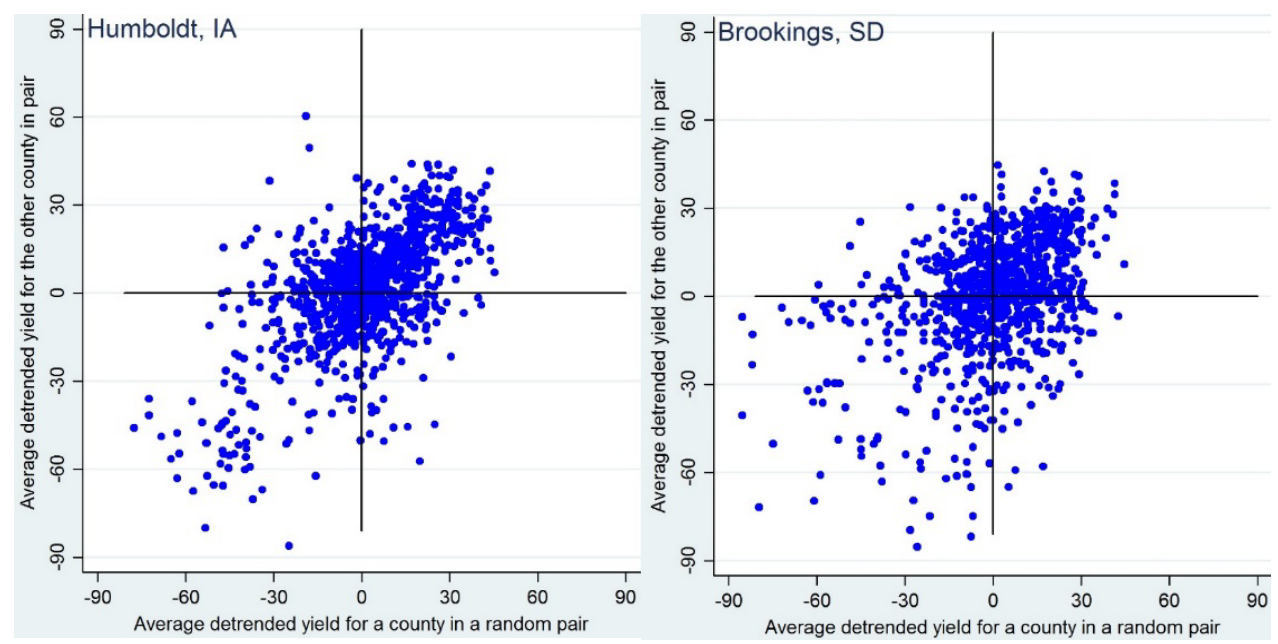

Note: The samples for each county in the figure are 50 randomly selected yield pairs, each of which has up to 20 years of average detrended yields.

Figure 7. For a County in Iowa and a County in South Dakota, Scatter Plot of Detrended Yields when Units are Assembled into Groups of 50, Averaged and then Randomly Matched with other Groups so Constructed

grouped units. This is the point that micro-correlation tends to strengthen upon aggregation, as explained in Cooke, Kousky and Joe (2011) and Hua (2012). The point is important because it demonstrates the limits of pooling risks within an insurance market.

Figure 8 considers sample counties in the two states. The figure shows within-year mean deviation from trend across all observed units in a given county and also within-year standard deviation of observed units. For Iowa, the six counties are Calhoun, Pocahontas, Webster, Humboldt, Wright and Hamilton. For South Dakota we use a total of six counties in the Central \{Sully, Hughes, Hyde, Hand, Beadle\} and East Central \{Brookings\} crop reporting regions. We use these counties for the sake of consistency as we also use them in an empirical assessment to be discussed later. For Iowa, the figure shows that yields within a county vary more in bad years than in good years. Some units hold up well, but others do not when stressed. For South Dakota, the pattern is very different in that yield variance among units in a county in a good year is larger than yield variance among units in a county in a bad year. Referring back to Figure 5, even in generally good years it appears that South Dakota yields are very sensitive to conditions 




Figure 8. The Average and Standard Deviation of Unit Observation Detrended Yields for Given Years within a County for Sample Counties in Iowa and in South Dakota

regardless of how county average yields compare with trend yields, whereas Iowa yields can be very stable when county average yields are at or above trend.

\section{Empirical Methods}

We apply five types of empirical methods to assess tail dependence among corn yield distributions at the farm level of aggregation. The first three assessments, discussed below, are A1) tail monotonicity statistics; A2) Kendall's $W$ statistics, and A3) goodness-of-fit tests for the Gaussian copula. In light of findings in A3), we also consider A4) exceedance correlation and symmetry tests, and A5) quantile and tail dependence tests.

A1) Tail monotonicity: In regard to relations (1)-(2), conditional probabilities $P\left[x_{2} \leq \hat{x}_{2} \mid x_{1} \leq \hat{x}_{1}\right]$ and $P\left[x_{2} \geq \hat{x}_{2} \mid x_{1} \geq \hat{x}_{1}\right]$ are calculated as follows:

(i) For individual insurance units in a chosen county, check for unit pairs that have corn yield records in ten common years, which may not be consecutive. 
(ii) Detrend the county unit yield following the procedure in Claassen and Just (2011), as outlined earlier.

(iii) For a matched unit pair, pick one (or other) unit and rank the detrended yield from the most negative to the most positive. This will be the $x_{1}$ (or $x_{2}$ ).

(iv) Calculate $P\left[x_{2} \leq \hat{x}_{2} \mid x_{1} \leq \hat{x}_{1}\right]$ and $P\left[x_{2} \geq \hat{x}_{2} \mid x_{1} \geq \hat{x}_{1}\right]$ for each matched pair using all yield records for $x_{1}$. Quantile value $q$ is chosen as from the set $\{0.1,0.2, \ldots, 0.9,1\}$.

(v) To check for robustness we drop the least negative $x_{1}$ and repeat step (iv).

(vi) Repeat step (v) until the set of common years is 4 .

(vii) As a further check on robustness, repeat (v)-(vi) by dropping the yield observations in the 'best' and 'worst' years, where 'best' and 'worst' are determined based on annual average yield of all samples in a county.

A2) Kendall's $W$ : Kendall's $W$ is a non-parametric concordance statistic (Kendall and Smith 1939; Legendre 2005; and Gibbons and Chakraborti 2010). We apply it to the insurance unit yields with exactly the same 10-years on record. The detrended yield for the ten common years are ranked from high through low. The sum of ranks for year $i(i \in\{1, \ldots, n\}$; $n=10)$ is $r_{i}=\sum_{j=1}^{m} r_{i, j}$, where $j \in\{1, \ldots, m\}$ denotes the unit yield record with $m$ observations in a chosen county. The mean of total ranks can be written as $\bar{r}=0.5 m(n+1)$. Kendall's $W$ is defined as

(28) $W=\frac{12 \sum_{i=1}^{n}\left(r_{i}-\bar{r}\right)^{2}}{m^{2} n\left(n^{2}-1\right)}$.

It can be readily shown that $W=1$ whenever there is complete consensus in rankings. Alternatively, $W=0$ implies $r_{i}=\bar{r} \forall i \in\{1, \ldots, n\}$, so that rankings are essentially random as far as they are intended to indicate common agreement. 
A3) Goodness-of-fit test for Gaussian copula: In recent years various procedures have emerged in the literature to test for goodness of fit for particular copulas. Genest, Rémillard and Beaudoin (2009) provide a detailed review of the literature. The test we apply in this study is commonly referred to as the 'blanket test,' where such non-parametric tests are not specific to a reference copula and limit the need for the analyst to make subjective modeling choices in regard to data categorization and parameter choices.

The cumulative distribution function of a two-dimensional random vector $\left(X_{1}, X_{2}\right)$ can be expressed by a copula model $C\left[F_{1}\left(x_{1}\right), F_{2}\left(x_{2}\right)\right]$, where $F_{1}(\cdot)$ and $F_{2}(\cdot)$ are univariate distributions of $X_{1}$ and $X_{2}$, respectively, and copula $C[\cdot]$ describes the specific dependence structure between the individual random variables. The null hypothesis is that copula $C[\cdot]$ belongs to some class $C_{0}[\cdot]$ (i.e., $H_{0}: C[\cdot] \in C_{0}[\cdot]$ ). The applied hypothesis test, or goodness-offit test, of $H_{0}$ is based on transformed ranks of observations, $U_{1}=\left(U_{1,1}, \ldots, U_{1, n}\right)$ and $U_{2}=$ $\left(U_{2,1}, \ldots, U_{2, n}\right)$, with $U_{j, i}$ being rescaled ranks for $n$ observations of $X_{i}$ (i.e., $U_{j, i}=R_{j, i} /(n+1)$ $\left.=n F_{j}\left(X_{j, i}\right) /(n+1)\right)$, where $F_{j}(\cdot)$ is the empirical cumulative distribution function computed from $X_{j}$. The empirical distribution of $\left(U_{1}, U_{2}\right)$ is defined as $C_{n}[\cdot]=$ $n^{-1} \sum_{i=1}^{n} 1\left\{U_{1, i} \leq u_{1}, U_{2, i} \leq u_{2}\right\}$, where $1\{\cdot\}$ is the set indicator function. ${ }^{6}$ The goodness-of-fit test is a Chi-square type test to compare the distance between $C_{n}[\cdot]$ and $C_{\theta}[\cdot]$, the latter being the estimated $C[\cdot]$ under the null hypothesis. The result is summarized by the Cramer-von Mises statistic $S_{n}$ and the associated $p$-value is approximated from the limiting distribution. ${ }^{7}$ Monte Carlo experiments indicate that the Cramer-von Mises statistic applied in this study is one of the

\footnotetext{
${ }^{6}$ See eqn. (1) in Genest, Rémillard and Beaudoin (2009).

${ }^{7}$ More detailed discussions can be found on pp. 200-201 in Genest, Rémillard and Beaudoin (2009).
} 
best goodness-of-fit test procedures for copula models (Genest, Rémillard and Beaudoin 2009).

A4) Exceedance correlation and symmetry test: Following Ang and Chen (2002) and Hong, Tu and Zhou (2007), the exceedance correlation between two groups of yields, $Y_{1}$ and $Y_{2}$, is calculated and correlation symmetry is tested. The exceedance correlation between two variables is defined as their correlation when both exceed a certain quantile level $c, 0<c<1$, of individual variables. Let $q\left(Y_{j}, c\right)$ denote the $c^{\text {th }}$ quantile of $Y_{j}$ with $j \in\{1,2\}$. The definition of exceedance correlation, when expressed mathematically, is: ${ }^{8}$

$$
\begin{aligned}
& \rho^{+}(c)=\operatorname{corr}\left(Y_{1}, Y_{2} \mid Y_{1}>q\left(Y_{1}, c\right), Y_{2}>q\left(Y_{2}, c\right)\right) ; \\
& \rho^{-}(c)=\operatorname{corr}\left(Y_{1}, Y_{2} \mid Y_{1}<q\left(Y_{1}, c\right), Y_{2}<q\left(Y_{2}, c\right)\right) .
\end{aligned}
$$

Our claim is that the left-tail correlation should be lower than the right-tail correlation in Iowa, while the reverse should be true in South Dakota. We will test whether symmetry fails.

The null hypothesis of the symmetry test is

$$
H_{0}: \rho^{+}(c)=\rho^{-}(c), \forall c \geq 0 .
$$

The test statistic $J_{p}$ developed in Hong, Tu and Zhou (2007, eq. 14, p. 1552) follows an asymptotic Chi-square distribution with degrees of freedom $m$, which is the chosen exceedance levels (number of quantiles) of $c$ in our case.

A5) Quantile and tail dependence: Following Patton (2012), for quantile $q, q \in(0,1)$, quantile dependence is defined as: ${ }^{9}$

\footnotetext{
8 These are eqns. (1)-(3) in Hong, Tu and Zhou (2007).

${ }^{9}$ These are eqns. (14)-(15) in Patton (2012).
} 


$$
\begin{aligned}
& \lambda^{q-}=P\left[U_{1, t} \leq q \mid U_{2, t} \leq q\right] \\
& \lambda^{q+}=P\left[U_{1, t}>q \mid U_{2, t}>q\right] \\
& \hat{\lambda}^{q-}=\frac{1}{T q} \sum_{t=1}^{T} 1\left\{U_{1, t} \leq q \mid U_{2, t} \leq q\right\} \\
& \hat{\lambda}^{q+}=\frac{1}{T(1-q)} \sum_{t=1}^{T} 1\left\{U_{1, t}>q \mid U_{2, t}>q\right\}
\end{aligned}
$$

Here $U_{j} \equiv F_{j}(\varepsilon)$ is the probability integral transformation of standardized yield residuals where the latter were generated by subtracting the mean from the yield residual and then dividing by the standard deviation. We use the empirical distribution function to non-parametrically estimate $F_{j}(\cdot)$ (i.e., $\left.\hat{F}_{j}(\varepsilon)=(T+1)^{-1} \sum_{t=1}^{T} 1\left\{\hat{\varepsilon}_{j t} \leq \varepsilon\right\}\right) .{ }^{10}$

As described in Patton (2012), tail dependence is defined as the limit of population quantile dependence as $q \rightarrow 0$ or $q \rightarrow 1,{ }^{11} \lambda^{L}=\lim _{q \downarrow 0} P\left[Y_{1, t} \leq q \mid Y_{2, t} \leq q\right]$ and $\lambda^{U}=$ $\lim _{q \uparrow 1} P\left[Y_{1, t}>q \mid Y_{2, t}>q\right]$. Upper and lower tail dependence are empirically estimated using the nonparametric method proposed in Frahm et al. (2005).

\section{Test Results and Analysis}

The test statistics for tail monotonicity, A1), are calculated based on matched unit pairs from the aforementioned yield records of ten common years in a county. Note that the record years are the same for each unit pair, but can vary across pairs. For Kendall's $W$, A2), we restrict the samples to be in the 10-year period that has the largest number of units (and observations) in a county.

The tail monotonicity test statistics for ten-year yield histories are reported in Table 1. They are calculated based on the matched unit pairs in Humboldt Co., Iowa and Brookings Co., South

${ }^{10}$ Division by $T+1$ rather than $T$ is well-accepted in the literature; see footnote 6 in Patton (2012).

${ }^{11}$ Eqn. (16) in Patton (2012). 
Table 1. Test Statistics for Tail Monotonicity (10-year yield record; Humboldt, IA and Brookings, South Dakota; Standard errors in the parentheses)

(a)

\begin{tabular}{|c|c|c|c|c|c|c|c|c|c|c|}
\hline \multicolumn{11}{|c|}{ LTD Statistic (Iowa) } \\
\hline$u / v$ & 0.1 & 0.2 & 0.3 & 0.4 & 0.5 & 0.6 & 0.7 & 0.8 & 0.9 & 1.0 \\
\hline \multirow[t]{2}{*}{0.1} & 0.24 & 0.42 & 0.56 & 0.67 & 0.76 & 0.83 & 0.90 & 0.94 & 0.98 & 1.00 \\
\hline & $(0.43)$ & $(0.49)$ & $(0.50)$ & $(0.47)$ & $(0.43)$ & $(0.37)$ & $(0.31)$ & $(0.23)$ & $(0.14)$ & \\
\hline \multirow[t]{2}{*}{0.2} & 0.21 & 0.39 & 0.52 & 0.64 & 0.73 & 0.81 & 0.88 & 0.93 & 0.98 & 1.00 \\
\hline & $(0.25)$ & $(0.31)$ & $(0.32)$ & $(0.32)$ & $(0.30)$ & $(0.26)$ & $(0.22)$ & $(0.17)$ & $(0.11)$ & \\
\hline \multirow[t]{2}{*}{0.3} & 0.18 & 0.35 & 0.48 & 0.61 & 0.71 & 0.79 & 0.87 & 0.93 & 0.97 & 1.00 \\
\hline & $(0.17)$ & $(0.22)$ & $(0.24)$ & $(0.24)$ & $(0.23)$ & $(0.21)$ & $(0.18)$ & $(0.14)$ & $(0.09)$ & \\
\hline \multirow[t]{2}{*}{0.4} & 0.16 & 0.32 & 0.45 & 0.57 & 0.68 & 0.77 & 0.85 & 0.92 & 0.97 & 1.00 \\
\hline & $(0.12)$ & $(0.16)$ & $(0.18)$ & $(0.19)$ & $(0.19)$ & $(0.18)$ & $(0.16)$ & $(0.13)$ & $(0.08)$ & \\
\hline \multirow[t]{2}{*}{0.5} & 0.15 & 0.29 & 0.42 & 0.54 & 0.65 & 0.75 & 0.84 & 0.91 & 0.97 & 1.00 \\
\hline & $(0.09)$ & $(0.12)$ & $(0.14)$ & $(0.15)$ & $(0.16)$ & $(0.15)$ & $(0.14)$ & $(0.11)$ & $(0.08)$ & \\
\hline \multirow[t]{2}{*}{0.6} & 0.14 & 0.27 & 0.39 & 0.51 & 0.62 & 0.73 & 0.82 & 0.89 & 0.96 & 1.00 \\
\hline & $(0.06)$ & $(0.09)$ & $(0.11)$ & $(0.12)$ & $(0.12)$ & $(0.12)$ & $(0.12)$ & $(0.10)$ & $(0.07)$ & \\
\hline \multirow[t]{2}{*}{0.7} & 0.13 & 0.25 & 0.37 & 0.49 & 0.60 & 0.70 & 0.80 & 0.88 & 0.95 & 1.00 \\
\hline & $(0.05)$ & $(0.07)$ & $(0.08)$ & (0.09) & (0.10) & $(0.10)$ & $(0.10)$ & $(0.09)$ & $(0.07)$ & \\
\hline \multirow[t]{2}{*}{0.8} & 0.12 & 0.23 & 0.35 & 0.46 & 0.57 & 0.67 & 0.77 & 0.86 & 0.94 & 1.00 \\
\hline & $(0.03)$ & $(0.05)$ & $(0.06)$ & $(0.06)$ & $(0.07)$ & $(0.08)$ & $(0.08)$ & $(0.08)$ & $(0.06)$ & \\
\hline \multirow[t]{2}{*}{0.9} & 0.11 & 0.22 & 0.32 & 0.43 & 0.54 & 0.64 & 0.74 & 0.84 & 0.93 & 1.00 \\
\hline & $(0.02)$ & $(0.03)$ & $(0.03)$ & $(0.04)$ & $(0.04)$ & $(0.05)$ & $(0.05)$ & $(0.06)$ & $(0.05)$ & \\
\hline 1.0 & 0.10 & 0.20 & 0.30 & 0.40 & 0.50 & 0.60 & 0.70 & 0.80 & 0.90 & 1.00 \\
\hline \multicolumn{11}{|c|}{ LTD Statistic (South Dakota) } \\
\hline \multirow[t]{2}{*}{0.1} & 0.15 & 0.28 & 0.40 & 0.51 & 0.61 & 0.70 & 0.80 & 0.88 & 0.95 & 1.00 \\
\hline & $(0.36)$ & $(0.45)$ & (0.49) & $(0.50)$ & (0.49) & $(0.46)$ & $(0.40)$ & $(0.33)$ & $(0.22)$ & \\
\hline \multirow[t]{2}{*}{0.2} & 0.14 & 0.27 & 0.38 & 0.50 & 0.60 & 0.70 & 0.79 & 0.87 & 0.95 & 1.00 \\
\hline & $(0.22)$ & $(0.29)$ & $(0.32)$ & $(0.33)$ & $(0.33)$ & $(0.31)$ & $(0.28)$ & $(0.23)$ & $(0.15)$ & \\
\hline \multirow[t]{2}{*}{0.3} & 0.13 & 0.25 & 0.37 & 0.48 & 0.58 & 0.69 & 0.78 & 0.87 & 0.94 & 1.00 \\
\hline & $(0.16)$ & $(0.21)$ & $(0.24)$ & $(0.26)$ & $(0.25)$ & $(0.24)$ & $(0.22)$ & $(0.18)$ & $(0.13)$ & \\
\hline \multirow[t]{2}{*}{0.4} & 0.12 & 0.25 & 0.36 & 0.47 & 0.57 & 0.67 & 0.77 & 0.86 & 0.94 & 1.00 \\
\hline & $(0.13)$ & $(0.17)$ & (0.19) & $(0.20)$ & $(0.21)$ & $(0.20)$ & $(0.18)$ & $(0.15)$ & $(0.11)$ & \\
\hline \multirow[t]{2}{*}{0.5} & 0.12 & 0.24 & 0.35 & 0.46 & 0.56 & 0.66 & 0.76 & 0.85 & 0.94 & 1.00 \\
\hline & $(0.10)$ & (0.13) & $(0.15)$ & $(0.16)$ & (0.17) & $(0.16)$ & $(0.15)$ & $(0.13)$ & $(0.09)$ & \\
\hline \multirow[t]{2}{*}{0.6} & 0.11 & 0.23 & 0.34 & 0.45 & 0.55 & 0.66 & 0.76 & 0.85 & 0.93 & 1.00 \\
\hline & $(0.08)$ & (0.11) & $(0.12)$ & $(0.13)$ & (0.13) & $(0.13)$ & $(0.13)$ & $(0.11)$ & $(0.08)$ & \\
\hline \multirow[t]{2}{*}{0.7} & 0.11 & 0.22 & 0.33 & 0.44 & 0.55 & 0.65 & 0.75 & 0.84 & 0.93 & 1.00 \\
\hline & $(0.06)$ & $(0.08)$ & $(0.09)$ & $(0.10)$ & (0.11) & $(0.11)$ & $(0.10)$ & $(0.09)$ & $(0.07)$ & \\
\hline \multirow[t]{2}{*}{0.8} & 0.11 & 0.22 & 0.32 & 0.43 & 0.53 & 0.64 & 0.74 & 0.83 & 0.92 & 1.00 \\
\hline & $(0.04)$ & $(0.06)$ & $(0.07)$ & $(0.07)$ & $(0.08)$ & $(0.08)$ & $(0.08)$ & $(0.07)$ & $(0.06)$ & \\
\hline \multirow[t]{2}{*}{0.9} & 0.11 & 0.21 & 0.32 & 0.42 & 0.52 & 0.62 & 0.72 & 0.82 & 0.92 & 1.00 \\
\hline & $(0.02)$ & $(0.03)$ & $(0.04)$ & $(0.05)$ & $(0.05)$ & $(0.05)$ & $(0.06)$ & $(0.05)$ & $(0.05)$ & \\
\hline 1.0 & 0.10 & 0.20 & 0.30 & 0.40 & 0.50 & 0.60 & 0.70 & 0.80 & 0.90 & 1.00 \\
\hline
\end{tabular}


Table 1. Continued

\begin{tabular}{|c|c|c|c|c|c|c|c|c|c|c|}
\hline \multicolumn{11}{|c|}{ RTI Statistic (Iowa) } \\
\hline$u / v$ & 0.1 & 0.2 & 0.3 & 0.4 & 0.5 & 0.6 & 0.7 & 0.8 & 0.9 & 1.0 \\
\hline 0.1 & 1.00 & 0.90 & 0.80 & 0.70 & 0.60 & 0.50 & 0.40 & 0.30 & 0.20 & 0.10 \\
\hline \multirow[t]{2}{*}{0.2} & 1.00 & 0.92 & 0.82 & 0.73 & 0.63 & 0.53 & 0.43 & 0.32 & 0.22 & 0.11 \\
\hline & & $(0.05)$ & $(0.05)$ & $(0.05)$ & $(0.05)$ & $(0.05)$ & $(0.04)$ & $(0.03)$ & $(0.03)$ & $(0.02)$ \\
\hline \multirow[t]{2}{*}{0.3} & 1.00 & 0.93 & 0.85 & 0.76 & 0.66 & 0.56 & 0.45 & 0.35 & 0.23 & 0.12 \\
\hline & & $(0.06)$ & $(0.08)$ & $(0.08)$ & $(0.08)$ & $(0.07)$ & $(0.07)$ & $(0.06)$ & $(0.04)$ & $(0.03)$ \\
\hline \multirow[t]{2}{*}{0.4} & 1.00 & 0.94 & 0.86 & 0.78 & 0.69 & 0.59 & 0.48 & 0.37 & 0.25 & 0.13 \\
\hline & & $(0.07)$ & $(0.09)$ & $(0.10)$ & $(0.10)$ & $(0.10)$ & $(0.09)$ & $(0.08)$ & $(0.06)$ & $(0.04)$ \\
\hline \multirow{2}{*}{0.5} & 1.00 & 0.94 & 0.88 & 0.80 & 0.72 & 0.62 & 0.52 & 0.40 & 0.28 & 0.15 \\
\hline & & $(0.08)$ & $(0.11)$ & $(0.12)$ & $(0.13)$ & $(0.13)$ & $(0.12)$ & $(0.11)$ & $(0.08)$ & $(0.05)$ \\
\hline \multirow[t]{2}{*}{0.6} & 1.00 & 0.95 & 0.89 & 0.82 & 0.74 & 0.65 & 0.55 & 0.44 & 0.31 & 0.17 \\
\hline & & $(0.09)$ & $(0.12)$ & $(0.14)$ & $(0.15)$ & $(0.16)$ & $(0.15)$ & $(0.14)$ & $(0.11)$ & $(0.08)$ \\
\hline \multirow[t]{2}{*}{0.7} & 1.00 & 0.96 & 0.90 & 0.84 & 0.77 & 0.69 & 0.59 & 0.47 & 0.34 & 0.19 \\
\hline & & $(0.10)$ & $(0.13)$ & $(0.16)$ & $(0.19)$ & $(0.19)$ & $(0.19)$ & $(0.18)$ & $(0.16)$ & $(0.11)$ \\
\hline \multirow[t]{2}{*}{0.8} & 1.00 & 0.96 & 0.92 & 0.86 & 0.80 & 0.72 & 0.63 & 0.52 & 0.39 & 0.22 \\
\hline & & $(0.11)$ & $(0.15)$ & $(0.18)$ & $(0.21)$ & $(0.23)$ & $(0.24)$ & $(0.23)$ & $(0.22)$ & $(0.16)$ \\
\hline \multirow[t]{2}{*}{0.9} & 1.00 & 0.96 & 0.92 & 0.88 & 0.83 & 0.76 & 0.68 & 0.57 & 0.44 & 0.27 \\
\hline & & $(0.13)$ & $(0.18)$ & $(0.22)$ & $(0.26)$ & $(0.29)$ & $(0.31)$ & $(0.32)$ & $(0.31)$ & $(0.25)$ \\
\hline \multirow{2}{*}{1.0} & 1.00 & 0.98 & 0.95 & 0.91 & 0.88 & 0.82 & 0.75 & 0.67 & 0.54 & 0.35 \\
\hline & & $(0.15)$ & $(0.23)$ & $(0.28)$ & $(0.33)$ & $(0.38)$ & $(0.43)$ & $(0.47)$ & $(0.50)$ & $(0.48)$ \\
\hline \multicolumn{11}{|c|}{ RTI Statistic (South Dakota) } \\
\hline 0.1 & 1.00 & 0.90 & 0.80 & 0.70 & 0.60 & 0.50 & 0.40 & 0.30 & 0.20 & 0.10 \\
\hline \multirow[t]{2}{*}{0.2} & 1.00 & 0.91 & 0.81 & 0.71 & 0.61 & 0.51 & 0.41 & 0.31 & 0.21 & 0.11 \\
\hline & & $(0.04)$ & $(0.05)$ & $(0.05)$ & $(0.06)$ & $(0.05)$ & $(0.05)$ & $(0.04)$ & $(0.04)$ & $(0.02)$ \\
\hline \multirow[t]{2}{*}{0.3} & 1.00 & 0.91 & 0.82 & 0.72 & 0.62 & 0.52 & 0.42 & 0.32 & 0.22 & 0.11 \\
\hline & & $(0.06)$ & $(0.07)$ & $(0.08)$ & $(0.08)$ & $(0.08)$ & $(0.08)$ & $(0.07)$ & $(0.06)$ & $(0.04)$ \\
\hline \multirow{2}{*}{0.4} & 1.00 & 0.91 & 0.82 & 0.73 & 0.64 & 0.54 & 0.44 & 0.33 & 0.23 & 0.12 \\
\hline & & $(0.07)$ & $(0.09)$ & $(0.10)$ & $(0.11)$ & $(0.11)$ & $(0.10)$ & $(0.09)$ & $(0.08)$ & $(0.05)$ \\
\hline \multirow[t]{2}{*}{0.5} & 1.00 & 0.91 & 0.83 & 0.74 & 0.65 & 0.55 & 0.45 & 0.35 & 0.24 & 0.12 \\
\hline & & $(0.08)$ & $(0.11)$ & $(0.13)$ & $(0.14)$ & $(0.14)$ & $(0.13)$ & $(0.12)$ & $(0.10)$ & $(0.07)$ \\
\hline \multirow[t]{2}{*}{0.6} & 1.00 & 0.92 & 0.84 & 0.75 & 0.66 & 0.56 & 0.46 & 0.36 & 0.25 & 0.14 \\
\hline & & $(0.10)$ & $(0.13)$ & $(0.15)$ & $(0.16)$ & $(0.17)$ & $(0.16)$ & $(0.15)$ & (0.13) & $(0.09)$ \\
\hline \multirow[t]{2}{*}{0.7} & 1.00 & 0.92 & 0.85 & 0.76 & 0.68 & 0.58 & 0.48 & 0.38 & 0.27 & 0.15 \\
\hline & & $(0.12)$ & $(0.16)$ & $(0.18)$ & $(0.20)$ & $(0.20)$ & $(0.20)$ & (0.19) & $(0.16)$ & $(0.12)$ \\
\hline \multirow[t]{2}{*}{0.8} & 1.00 & 0.93 & 0.86 & 0.78 & 0.70 & 0.61 & 0.51 & 0.41 & 0.30 & 0.17 \\
\hline & & $(0.14)$ & $(0.19)$ & $(0.22)$ & $(0.24)$ & $(0.25)$ & $(0.25)$ & $(0.24)$ & $(0.21)$ & (0.17) \\
\hline \multirow{2}{*}{0.9} & 1.00 & 0.93 & 0.87 & 0.80 & 0.72 & 0.63 & 0.54 & 0.44 & 0.33 & 0.20 \\
\hline & & $(0.17)$ & $(0.23)$ & $(0.27)$ & $(0.30)$ & $(0.32)$ & (0.33) & $(0.32)$ & $(0.30)$ & $(0.24)$ \\
\hline \multirow[t]{2}{*}{1.0} & 1.00 & 0.95 & 0.90 & 0.84 & 0.77 & 0.69 & 0.61 & 0.51 & 0.40 & 0.25 \\
\hline & & $(0.22)$ & $(0.30)$ & $(0.37)$ & $(0.42)$ & $(0.46)$ & $(0.49)$ & $(0.47)$ & $(0.49)$ & $(0.43)$ \\
\hline
\end{tabular}


Dakota. ${ }^{12}$ Now consider one column for the LTD statistic in panel (a), say $v=0.5$. Moving down the column we see that the probability that $x_{2}$ is below the unconditional median, 0.5 , generally declines as $u$ increases. Unconditionally (i.e., when $u=1$ ), the probability is of course 0.5. However, when the upper bound condition on $x_{1}$ (i.e., on $u$ ) falls, then the probability grows. This indicates that the two sets of detrended yields bunch together in the tail in the left-tail decreasing sense. Panel (b) of Table 1 shows a similar pattern in the RTI statistic. In summary, Table 1 provides evidence of left-tail decreasing and right-tail increasing behavior between unit pairs in the chosen county. Therefore, as to confirm robustness, the results for fewer sample years after deleting the best or worst years were also calculated. Patterns, reported in Appendix B, are consistent with those described above.

Table 2 presents the results for Kendall's $W$ in Humboldt Co., Iowa and Brookings, South Dakota. The statistic is calculated based on all units with a 10-year detrended yield record over 1999-2008. The first row reports the statistics after deleting yield record for the best years except that for the ten year record (0.42), while the second row reports the statistics after deleting the worst years gradually. We see that, in general, Kendall’s $W$ decreases when more years are deleted. This means that upon moving to the tails (right or left), ranks of the

Table 2. Kendall's W Statistic for Humboldt, Iowa and Brookings, South Dakota

\begin{tabular}{lrrrrrrr}
\hline Years of yield record & 10 & 9 & 8 & 7 & 6 & 5 & 4 \\
\hline Iowa & & & & & & & \\
\hline After deleting years with best yield & 0.42 & 0.33 & 0.27 & 0.25 & 0.22 & 0.21 & 0.16 \\
After deleting years with worst yield & 0.42 & 0.42 & 0.35 & 0.30 & 0.26 & 0.26 & 0.23 \\
\hline South Dakota & & & & & & & \\
\hline After deleting years with best yield & 0.19 & 0.10 & 0.08 & 0.04 & 0.04 & 0.03 & 0.02 \\
After deleting years with worst yield & 0.19 & 0.18 & 0.19 & 0.18 & 0.16 & 0.17 & 0.17 \\
\hline
\end{tabular}

12 These two counties belong to the county groups in Iowa and South Dakota that we use for the empirical tests A4) and A5). The results are very similar to other proximate counties in Iowa and South Dakota. 
detrended yields become increasingly concentrated across units (i.e., there is greater yield dependence in the tails). The statistics for other counties across states, which are reported in Appendix B, confirm a similar pattern.

Empirical analyses of A3) Gaussian goodness-of-fit test, A4) exceedance correlation/symmetry test and A5) quantile/tail dependence test described above focus on the upper and lower tails dependence structures for two yield series in each state and seek to compare Iowa with South Dakota. From the insurance unit-level detrended yield observations discussed in the previous data section, we construct two yield data series for each state. Individual data series are combined from a group of counties in the state in order to have sufficient sample units. For Iowa, the two groups are Group 1= county set \{Calhoun, Pocahontas, Webster $\}$ and Group $2=$ county set $\{$ Humboldt, Wright, Hamilton $\}$. These are adjacent county sets in North Central Iowa where Group 2 is slightly to the north and east of Group 1. In South Dakota, the two groups for comparison are based on crop reporting districts. Here Group 1 = county set $\{$ Sully, Hughes, Hyde, Hand, Beadle, Buffalo, Jerauld, Brule, Aurora\} comprising the Central crop reporting district while Group 2 = county set $\{$ Kingsbury, Brookings, Sanborn, Miner, Lake, Moody, Davison, Hanson, McCook, Minnehaha\} comprising the East Central reporting district. Detrended yield data in each group were treated as realizations from the same marginal distribution. Then 100,000 yield observations were sampled with replacement for each sample year and for both groups. The total sample size was 1 million for each yield data series. Summary statistics for the generated data are reported in the lower panel of Table 3.

For the following analyses, the yields in each group were standardized to have zero mean and unit standard deviation. For comparison, we also generated 10 million samples from the bivariate normal distribution with the same unconditional correlation as the pair of yield data 
Table 3. Summary Statistics of the Detrended Yield Data used for A3, A4 and A5

\begin{tabular}{|c|c|c|c|c|c|}
\hline State & Group & Mean & S.D. & Min & Max \\
\hline \multicolumn{6}{|c|}{ For A3 } \\
\hline \multirow[t]{2}{*}{ IA } & 1 & 0.17 & 21.46 & -175.96 & 77.50 \\
\hline & 2 & 0.68 & 20.69 & -157.46 & 83.70 \\
\hline \multirow[t]{2}{*}{ SD } & 1 & -0.42 & 37.69 & -119.35 & 119.61 \\
\hline & 2 & 0.36 & 26.94 & -149.85 & 172.41 \\
\hline \multicolumn{6}{|c|}{ For A4 and A5 } \\
\hline \multirow[t]{2}{*}{ IA } & 1 & 0.27 & 20.95 & -178.53 & 82.61 \\
\hline & 2 & 0.68 & 20.65 & -166.47 & 90.10 \\
\hline \multirow[t]{2}{*}{ SD } & 1 & -0.46 & 37.65 & -124.14 & 119.61 \\
\hline & 2 & 0.58 & 26.70 & -160.32 & 172.41 \\
\hline
\end{tabular}

series in each state. For Gaussian goodness-of-fit test A3), in order to save computation time for each state group we drew 1,000 samples without replacement for each year in the 10 year period 1999-2008. The exception was the Central group of South Dakota, for which we drew samples with replacement because only 623 units were observed in that region. Therefore, we have 10,000 observations for each group for the goodness-of-fit test, the summary statistics of which are contained in the upper panel of Table 3.

Gaussian copula goodness-of-fit test results are reported in Table $4 .{ }^{13}$ The test rejects the null hypothesis that the Gaussian copula fits well at significance levels lower than one percent, indicating that the Gaussian copula does not fit the data well. Correlation and dependence tests A4) and A5) are used to characterize the nature of this poor fit.

Table 4. Gaussian Goodness-of-Fit Tests Results

\begin{tabular}{lcc}
\hline & Iowa & South Dakota \\
\hline Test Statistic & 0.4616 & 0.4649 \\
p-value & 0.0005 & 0.0005 \\
\hline
\end{tabular}

${ }^{13}$ The goodness-of-fit test is done using the "copula" package in R. 
Figure 9 presents exceedance correlations among quantiles between the two series of yield data ${ }^{14}$ Iowa and South Dakota are in the upper and lower panels, respectively, where the $c$ values increase in 0.025 increments between 0.025 and 0.975 . The figure shows that, compared with the bivariate normal, Iowa yields tend to be more correlated on the upper tail, while South Dakota yields are more likely to move together in the lower tail. The null hypothesis of the

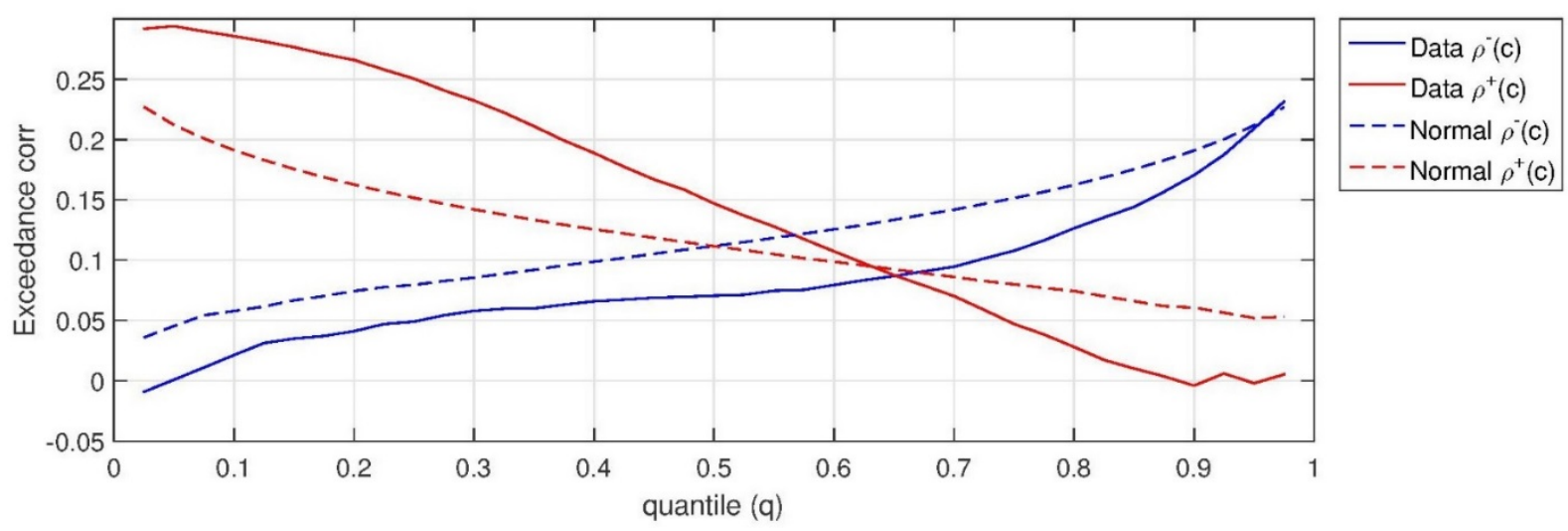

(a) Iowa

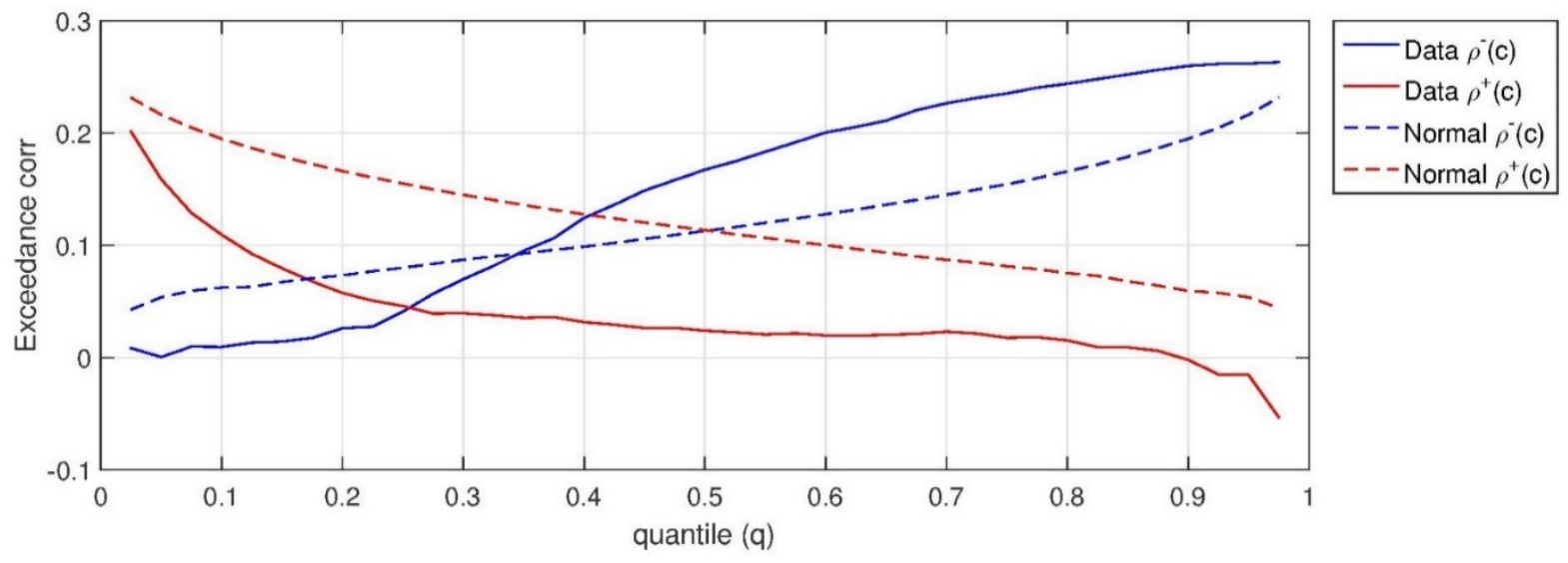

(b) South Dakota

Figure 9. Exceedance Correlations for Standardized Detrended Yields

${ }^{14}$ The calculations and tests for A4) and A5), including exceedance correlation/symmetry test and quantile/tail dependence as well as the associated figures (figures 9, 10, and 11) are arrived at by modifying the code accompanying Patton (2012), available at http://public.econ.duke.edu/ ap172/code.html. 
correlation symmetry is rejected for both Iowa and South Dakota with a p-value close to 0. The test statistics are 118.93 and 126.80 for Iowa and South Dakota, respectively.

Figure 10 presents the corresponding quantile dependences and the difference between the upper and lower tails in Iowa and South Dakota, together with those calculated for the simulated bivariate normal. All the quantile dependence and difference estimates are significant at the 10 percent level. The results indicate that, when compared with the bivariate normal benchmark dependence structure, Iowa corn yields are relatively more 'dependent' in the upper tail. This is consistent with Figure 5, as land quality heterogeneities matter only in the left tail for our stylized model of Iowa yields. By contrast, for South Dakota, the lower tail shows a higher level of dependence than the upper tail. This is also consistent with our Figure 5, as heterogeneities matter only in the right tail for our stylized model of South Dakota yields.

To further illustrate non-symmetric relations in yield data dependence structures, we patch the upper and lower tail dependence measures together at the median ( $q=0.5$ in eqn. (31)) and calculate the corresponding difference, In other words,

$$
\begin{aligned}
& \lambda^{q-}=P\left[U_{1, t} \leq q \mid U_{2, t} \leq q\right], 0<q \leq 0.5 ; \\
& \lambda^{q+}=P\left[U_{1, t}>q \mid U_{2, t}>q\right], 0.5<q<1 ; \\
& \hat{\lambda}^{q-}=\frac{1}{T q} \sum_{t=1}^{T} 1\left\{U_{1, t} \leq q \mid U_{2, t} \leq q\right\}, 0<q \leq 0.5 ; \\
& \hat{\lambda}^{q+}=\frac{1}{T(1-q)} \sum_{t=1}^{T} 1\left\{U_{1, t}>q \mid U_{2, t}>q\right\}, 0.5<q<1 ;
\end{aligned}
$$

The results are reported in Figure 11, which presents the pointwise differences in quantile dependence and the bootstrapped 90 percent confidence intervals. We see that for the variables simulated from the bivariate normal, the difference is a constant, zero. For Iowa, the difference between the upper and lower tails are all positive, meaning that the upper tail shows stronger dependence than the lower tail. For South Dakota, the difference is uniformly negative, which is consistent with greater lower tail dependence. 

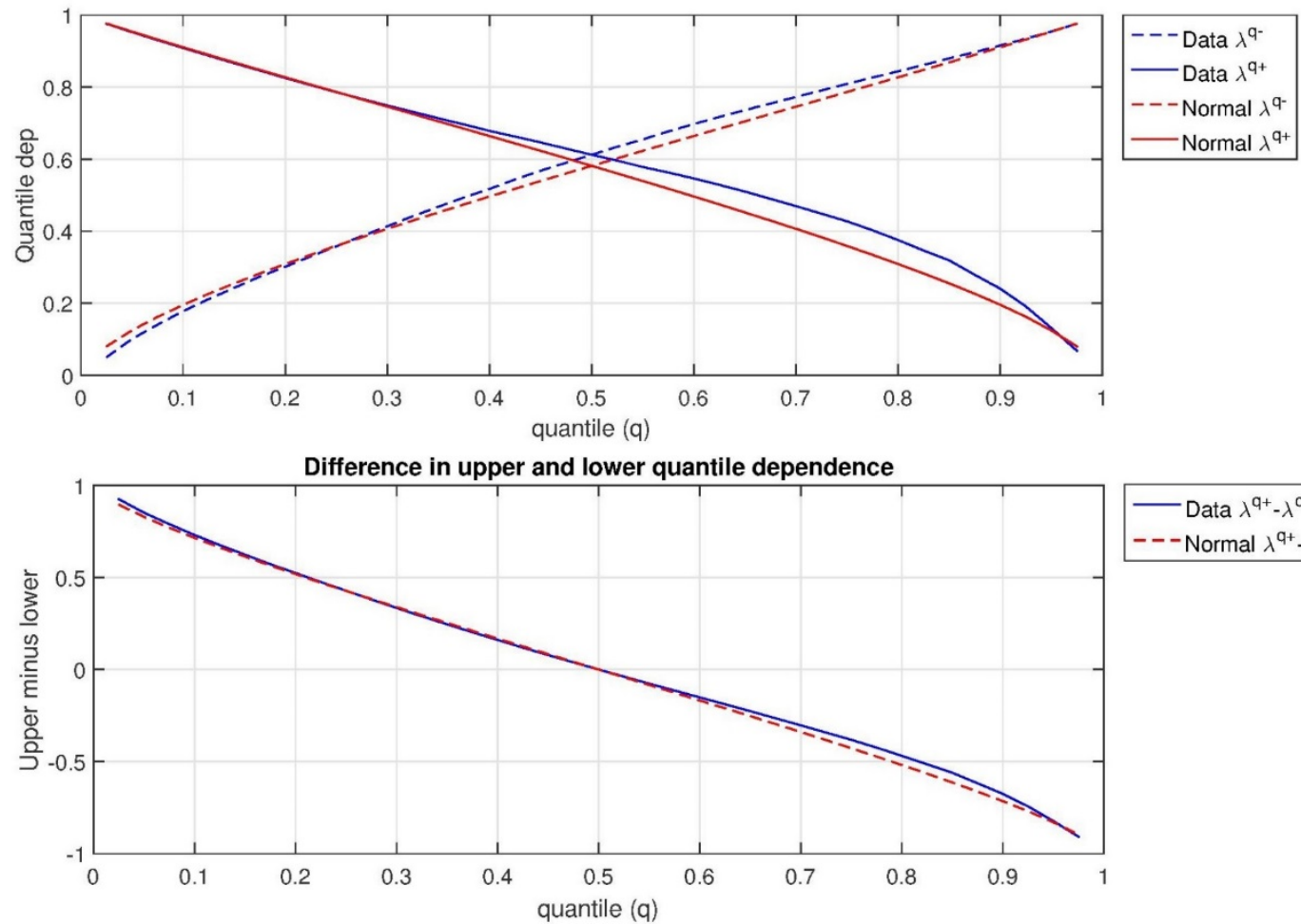

$$
\begin{aligned}
& \text { Data } \lambda^{q^{+}}-\lambda^{q^{-}} \\
& --- \text {Normal } \lambda^{q^{+}}-\lambda^{q^{-}}
\end{aligned}
$$

(a) Iowa
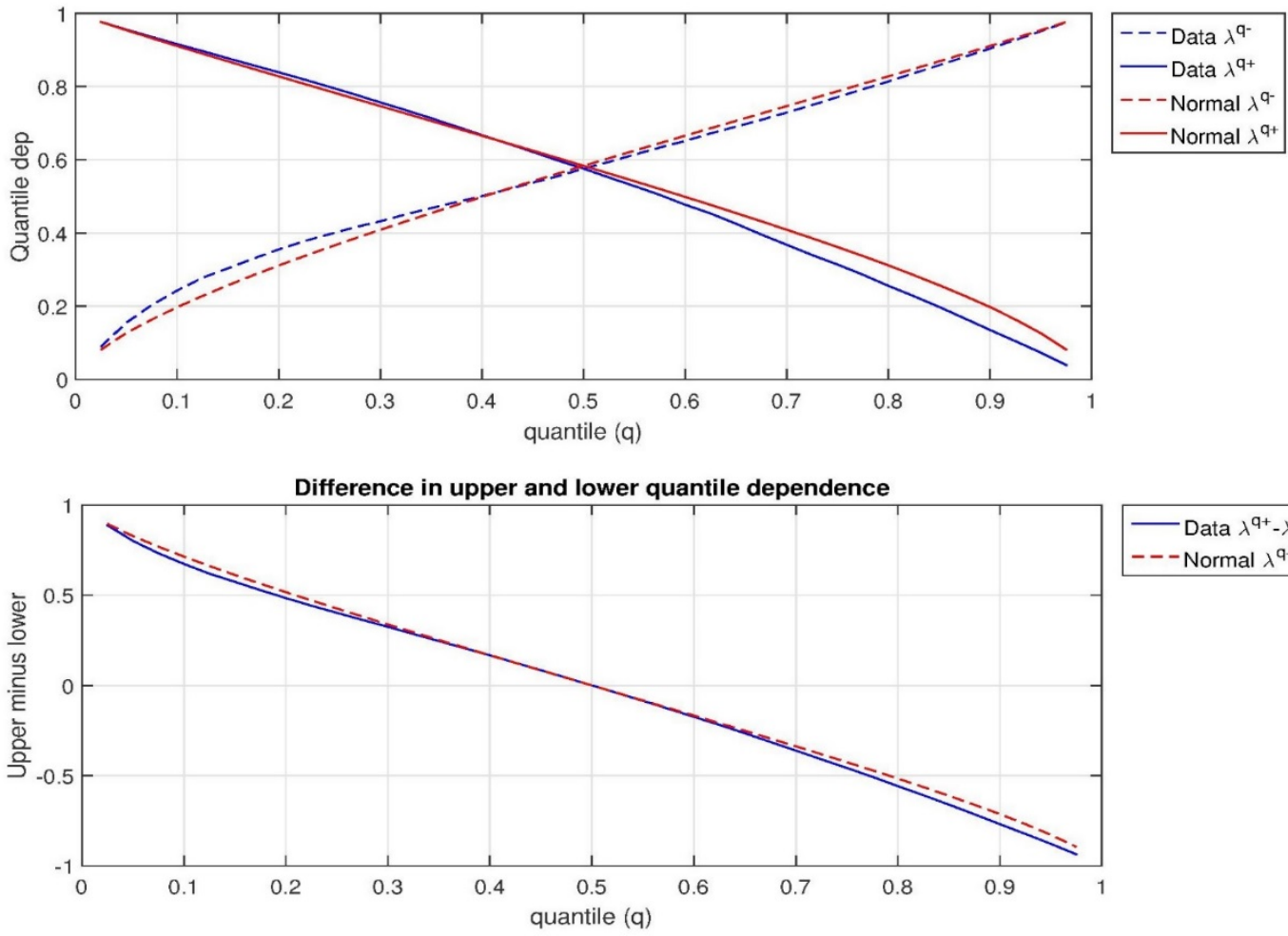

Data $\lambda^{q+}-\lambda^{q^{-}}$
--- Normal $\lambda^{q^{+}}-\lambda^{q^{-}}$

(b) South Dakota

Figure 10. Quantile Dependence and Upper-Lower Differences 

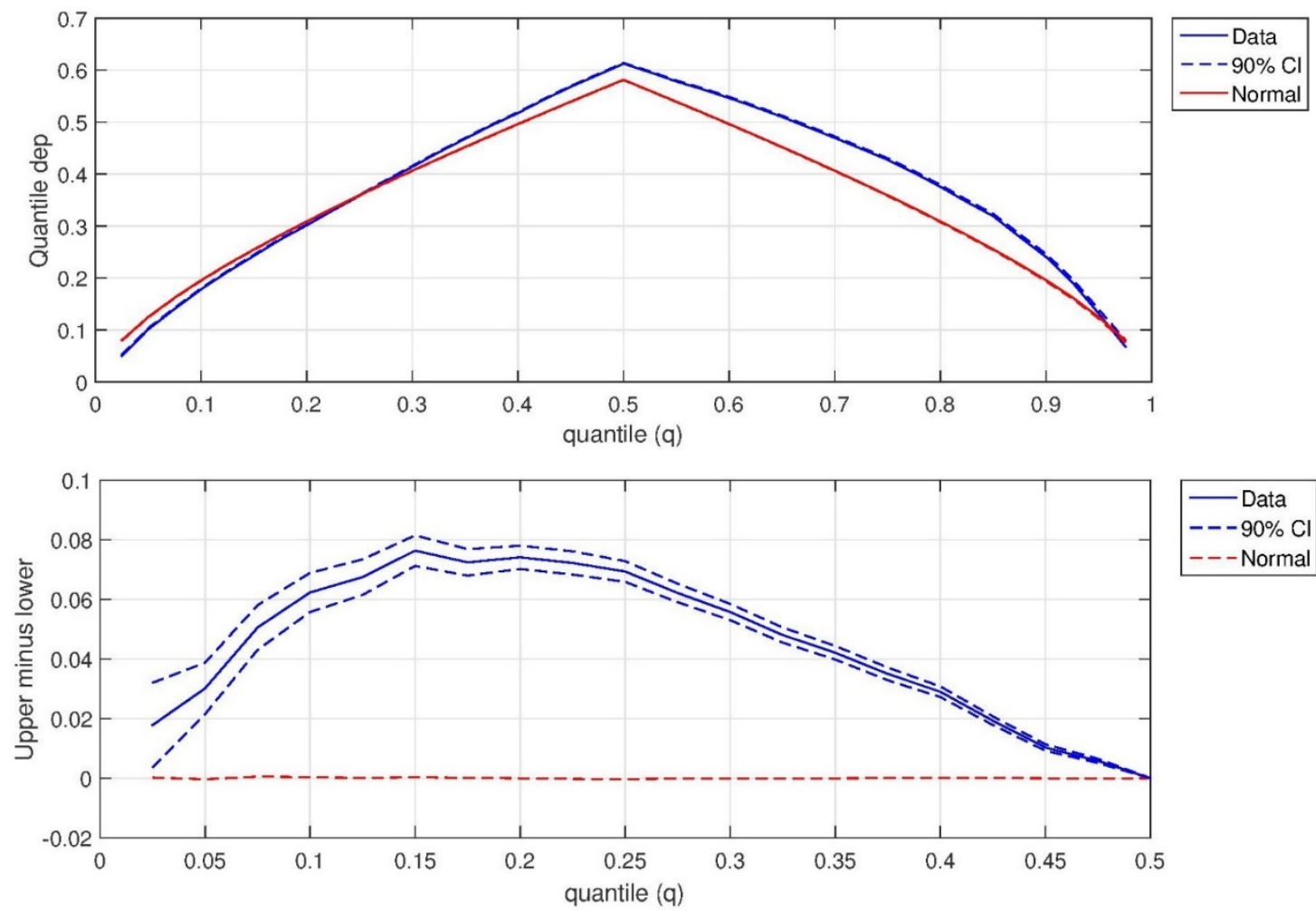

- Data $---90 \% \mathrm{Cl}$ --- Normal

(a) Iowa
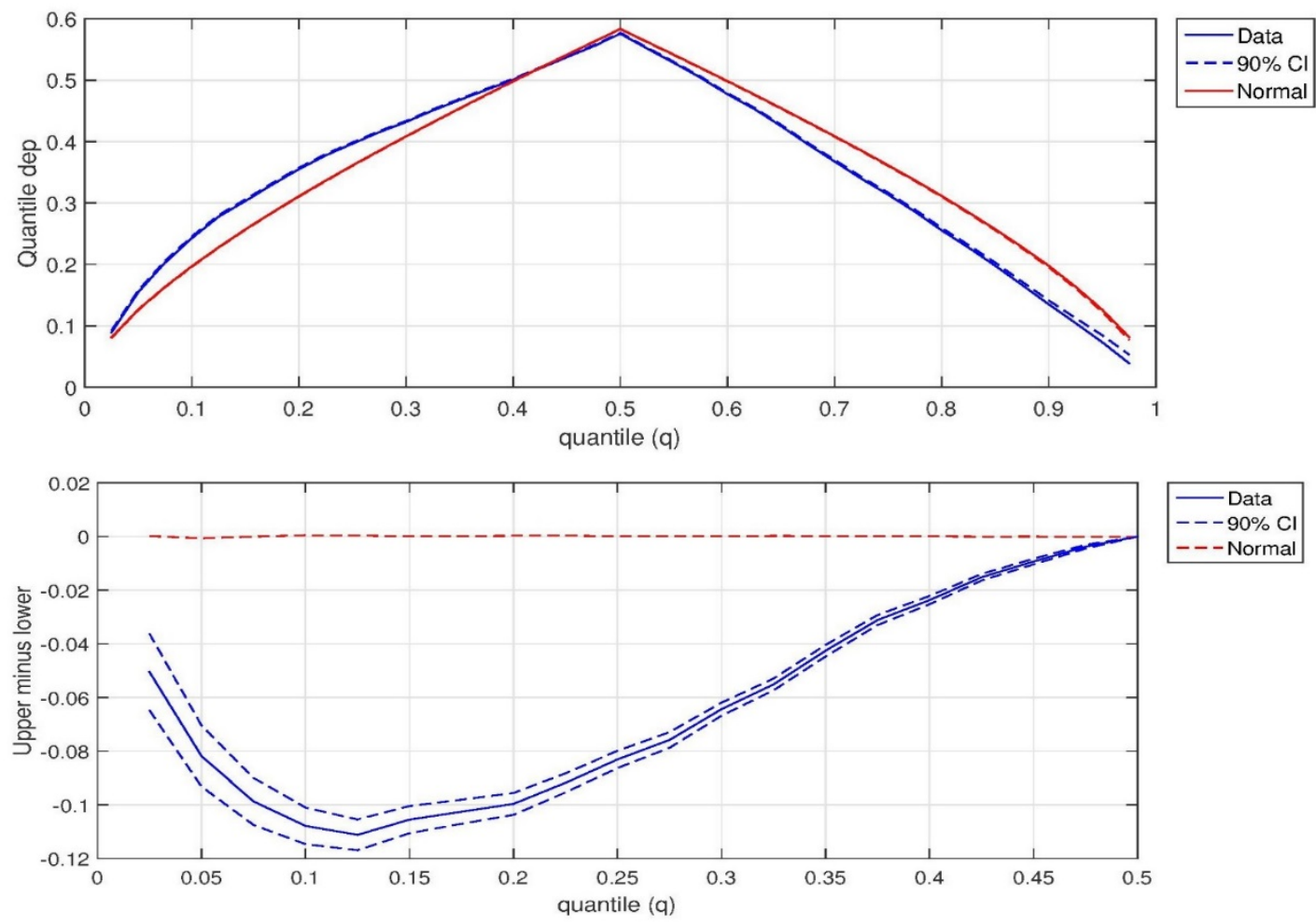

$--90 \% \mathrm{Cl}$ --- Normal

(b) South Dakota

Figure 11. Difference in Upper and Lower Quantile Dependences 
For Iowa, the non-parametrically estimated lower tail dependence coefficient is 0.0044 with 90 percent bootstrap confidence interval [0, 0.0336]. The upper tail dependence coefficient is 0.0081 with confidence interval [0, 0.0390]. Thus, we fail to reject the hypothesis of zero tail dependence in both tails. For South Dakota, the lower tail dependence is estimated as 0.012 with confidence interval $[0.0009,0.045]$ while the upper tail dependence is 0.0002 with confidence interval [0, 0.0282]. The null hypothesis of zero tail dependence is rejected for the lower tail but not for the upper tail.

\section{Concluding Remarks}

In this paper, we have sought to do three things. We have developed a model that emphasizes the role of natural resources in determining the structure of yield-yield correlations in a geographic area. In particular the model connects what we refer to as land yield resilience with heterogeneities in land and weather endowments so that yield-yield correlation structures can be related to growing conditions in a given year. Secondly we have used this model to propose and then provide empirical evidence on some hypotheses. In particular, we have posited that if an area is such that crops tend to grow well then yield correlations should be larger when growing conditions are good than when they are poor. Conversely, if an area is such that crops do not tend to grow well, then yield correlations should be larger when growing conditions are poor than when they are good. A further implication of the above is that the Gaussian copula, currently an implicit modeling assumption for crop insurance rate setting in the United States, should not provide a good fit for yield data. Our third contribution is to scrutinize yield-yield conditional correlation structures using yield data obtained from USDA Risk Management Agency data. We find evidence to support the inferences drawn from our land resilience model. In so doing, we cast doubt on the appropriateness of current Risk Management Agency rate setting methodologies.

Our general point that yield-yield correlations should vary with overall growing conditions 
also suggests caution when imposing constant price-yield correlation structures (whether cardinal or rank correlations), as is done when modeling revenue insurance (Mildenhall 2005;

Coble et al. 2010). Gustafson’s (1958) seminal work on how crop carryover affects commodity markets has led to robust insights on price formation for storable commodities. In particular, Deaton and Laroque (1992, theorem 1) demonstrates that price movements should be more sensitive to shocks when stocks are low than when they are high. The current year's price should be determined only by current demand when stocks in storage are low whereas current and future year markets should be coupled when substantive storage is likely to take place across harvest. Thus, the correlation between price and aggregate yield will depend only on supply and demand shocks in the current year when stocks are too low to buffer across years, but price will correlate with shocks that hold information on current year and future supply and demand when stocks are sufficient to provide meaningful buffering storage across harvest.

For this reason, price-yield correlations should be more negative when stocks are low and growing conditions ominous relative to when either stocks in storage are high or growing conditions are propitious. Thus, the natural price-yield hedge may strengthen when most needed. This conjecture may be more reassuring to revenue contract underwriters than our current finding that yield correlations strengthen in tails. In any case, modeling structures that force constant price-yield correlations are also suspect and warrant empirical scrutiny.

\section{References}

Aas, K. 2004. "Modelling the Dependence Structure of Financial Assets: A survey of four copulas.” Unpublished manuscript, Norwegian Computing Center, NR Note no. SAMBA/22/04.

Ang, A., and J. Chen. 2002. “Asymmetric Correlations of Equity Portfolios.” Journal of Financial Economics 63(3):443-494. 
Bagnoli, M., and T.C. Bergstrom. 2005. “Log-Concave Probability and its Applications.” Economic Theory 26(2):445-469.

Berck, P., and G. Helfand. 1990. "Reconciling the von Liebig and Differentiable Crop Production Functions.” American Journal of Agricultural Economics 72(4):985-996.

Berghaus, B., and A. Bücher. 2014. “Nonparametric Tests for Tail Monotonicity.” Journal of Econometrics 180(2):117-126.

Boyer, C.N., B.W. Brorsen, and E. Tumusiime. 2015. "Modeling Skewness with the Linear Stochastic Plateau Model to Determine Optimal Nitrogen Rates.” Agricultural Economics 46(1):1-10.

Calviño, P.A., and V.O. Sadras. 1999. "Interannual Variation in Soybean Yield: Interactions among rainfall, soil depth and crop management.” Field Crops Research 63(3):237-246.

Claassen, R., and R.E. Just. 2011. "Heterogeneity and Distributional Form of Farm-Level Yields.” American Journal of Agricultural Economics 93(1):144-160.

Coble, K.H., T.O. Knight, B.K. Goodwin, M.F. Miller, and R.M. Rejesus. 2010. A Comprehensive Review of the RMA APH and COMBO Rating Methodology Final Report 2010. A report completed by Sumaria Systems for the United States Department of Agriculture, Risk Management Agency. Available at http://www.rma.usda.gov/pubs/2009/comprehensivereview.pdf.

Cooke, R.M., C. Kousky, and H. Joe. 2011. “Micro Correlations and Tail Dependence.” In D. Kurowicka and H. Joe (eds.), Ch. 5, pp. 89-112, Dependence Modeling: Vine Copula Handbook. Singapore: World Scientific Publishing Co.

Deaton, A., and G. Laroque. 1992. “On the Behavior of Commodity Markets.” Review of Economic Studies 59(1):1-23.

Deelstra, G., J. Dhaene, and M. Vanmaele. 2011. “An Overview of Comonotonicity and Its Applications in Finance and Insurance.” In G. Di Nunno and B. Øksendal, eds. Advanced 
Mathematical Methods for Finance. Berlin: Springer-Verlag, pp. 155-179.

Du, X., D.A. Hennessy, and H. Feng. 2014. “A Natural Resource Theory of U.S. Crop Insurance Contract Choice.” American Journal of Agricultural Economics 96(1):232-251.

Du, X., D.A. Hennessy, and C. Yu. 2012. “Testing Day’s Conjecture that More Nitrogen Decreases Crop Yield Skewness.” American Journal of Agricultural Economics 94(1):225237.

Du, X., C.L. Yu, D.A. Hennessy, and R. Miao. 2015. “Geography of Crop Yield Skewness.” Agricultural Economics, forthcoming.

Durrett, R. 1996. Probability: Theory and Examples, $2^{\text {nd }}$ edn. Wadsworth Publishing Co.: Belmont CA.

Frahm, G., M. Junker, and R. Schmidt. 2005. “Estimating the Tail-dependence Coefficient: Properties and Pitfalls.” Insurance: Mathematics and Economics 37(1):80-100.

Genest, C., B. Rémillard, and D. Beaudoin. 2009. “Goodness-of-fit Tests for Copulas: A Review and a Power Study.” Insurance: Mathematics and Economics 44:199-213.

Gibbons, J. D., and S. Chakraborti. 2010. Nonparametric Statistical Inference. Dekker, New York.

Goodwin, B.K. 2001. "Problems with Market Insurance in Agriculture.” American Journal of Agricultural Economics 83(3):643-649.

Goodwin, B.K., and A. Hungerford. 2015. "Copula-Based Models of Systemic Risk in U.S. Agriculture: Implications for Crop Insurance and Reinsurance Contracts.” American Journal of Agricultural Economics 97(3):879-896.

Glauber, J.W. 2013. "The Growth of the Federal Crop Insurance Program, 1990-2011.” American Journal of Agricultural Economics 95(2):482-488.

Gustafson, R.L. 1958. Carryover Levels for Grains: A method for determining amounts that are optimal under specified conditions. U.S. Dept. of Agric. Technical Bulletin 1178, 
Washington, DC, October.

Hao, L., and D. Q. Naiman. 2007. Quantile Regression. Thousand Oaks, CA: Sage Publications.

Helms, D. 1992. “The Development of the Land Capability Classification.” In D. Helms (Ed.), pp. 60-73, Readings in the History of the Soil Conservation Service. Washington, DC: Soil Conservation Service http://www.nrcs.usda.gov/Internet/FSE_DOCUMENTS/stelprdb1043484.pdf

Hennessy, D.A. 2009. “Crop Yield Skewness and the Normal Distribution.” Journal of Agricultural and Resource Economics 34(1):34-52.

Hong, Y., J. Tu, and G. Zhou. 2007. “Asymmetries in Stock Returns: Statistical Tests and Economic Evaluation.” Review of Financial Studies 20(5):1547-1581.

Hua, L. 2012. Multivariate Extremal Dependence and Risk Measures. PhD Dissertation, Dept. of Statistics, University of British Columbia. Available at https://circle.ubc.ca/bitstream/handle/2429/42475/ubc_2012_fall_hua_lei.pdf?sequence=1.

Iman, R.L., and W.J. Conover. 1982. “A Distribution-free Approach to Inducing Rank Correlation among Input Variables.” Communications in Statistics Part B - Simulation and Computation 11(3):311-334.

Joe, H. 2015. Dependence Modeling with Copulas. CRC Press: Boca Raton FL.

Just, R.E., L. Calvin, and J. Quiggin. 1999. “Adverse Selection in Crop Insurance: Actuarial and Asymmetric Information Incentives.” American Journal of Agricultural Economics 81(4):834-849.

Kendall, M.G., and B.B. Smith. 1939. “The Problem of $m$ Rankings.” Annals of Mathematical Statistics 10(3):275-287.

Ketterings, Q., S. Reid, and R. Rao. 2007. “Cation Exchange Capacity (CEC).” Fact Sheet 22, Cornell University Cooperative Extension, New York, http://nmsp.cals.cornell.edu/publications/factsheets/factsheet22.pdf, visited 4/23/2015. 
Legendre, P. 2005. "Species Associations: The Kendall Coefficient of Concordance Revisited.” Journal of Agricultural, Biological, and Environmental Statistics 10(2):226-245.

Li, D.X. 2000. “On Default Correlation: A Copula Function Approach.” Journal of Fixed Income 9(4):43-54.

Mildenhall, S.J. 2005. “The Report of the Research Working Party on Correlations and Dependencies Among All Risk Sources: Part 1-Correlation and Aggregate Loss Distributions with an Emphasis on the Iman-Conover Method.” Research Working Party on Correlations and Dependencies Among All Risk Sources, Casualty Actuarial Society. Available at https://www.casact.org/research/index.cfm?fa=workingparty-corr.

Nelsen, R.B. 1999. An Introduction to Copulas. New York: Springer.

Patton, A.J. 2013. “Copula Methods for Forecasting Multivariate Time Series.” In G. Elliott and A. Timmermann (eds.), Ch. 16, pp. 899-960, Handbook of Economic Forecasting, Vol. 2, Part B. Amsterdam: North Holland.

Rudin, W. 1976. Principles of Mathematical Analysis, $3^{\text {rd }}$ edn. McGraw Hill: New York. Trivedi, P.K., and D.M. Zimmer. 2005. “Copula Modeling: An Introduction for Practitioners.” Foundations and Trends ${ }^{\circledR}$ in Econometrics 1(1):1-111.

Varian, H.R. 1992. Microeconomic Analysis, $3^{\text {rd }}$ edn. W.W. Norton \& Co.: New York.

Williams, C.L., M. Liebman, J.E. Edwards, D.E. James, J.W. Singer, R. Arritt, and D. Herzmann. 2008. "Patterns of Regional Yield Stability in Association with Regional Environmental Characteristics.” Crop Science 48(4):1545-1559.

Zimmer, D.M. 2012. “The Role of Copulas in the Housing Crisis.” Review of Economics and Statistics 94(2):607-620.

Zimmer, D.M. 2014. “Crop Price Comovements During Extreme Market Downturns.” Working paper, Dept. of Economics, Western Kentucky University, May 28. 


\section{Appendix A}

Define residual variability over the growing season by $w_{m}=\sum_{n=m}^{N} \omega_{n}$, so that, by expansion and use of independence, the conditional centralized third moment is

$$
\alpha_{m}=\mathbb{E}\left[w_{m}^{3}\right]=\mathbb{E}\left[\left(\sum_{n=m}^{N} \omega_{n}\right)^{3}\right]=R \mathbb{E}\left[\omega^{3}\right], \quad R \equiv N-m+1
$$

Similarly, one can compute the conditional uncentralized fourth moment as

$$
\begin{gathered}
\mathbb{E}\left[w_{m}^{4}\right]=\mathbb{E}\left[\left(\sum_{n=m}^{N} \omega_{n}\right)^{4}\right]=R \mathbb{E}\left[\omega_{m}^{4}\right]+\mathbb{E}\left[6 \sum_{i=m}^{N} \sum_{j=m}^{N} \omega_{i}^{2} \omega_{j}^{2}\right] \\
=R \mathbb{E}\left[\omega^{4}\right]+6 \sigma^{4} \mathbb{E}\left[\sum_{i=m}^{N} \sum_{j=m}^{N} 1\right]=R \mathbb{E}\left[\omega^{4}\right]+6 R^{2} \sigma^{4} .
\end{gathered}
$$

Therefore,

$$
\begin{aligned}
\beta_{m} & =\mathbb{E}\left[w_{m}^{4}\right]-\left(\mathbb{E}\left[w_{m}^{2}\right]-\left(\mathbb{E}\left[w_{m}\right]\right)^{2}\right)^{2}=\mathbb{E}\left[\left(\sum_{n=m}^{N} \omega_{n}\right)^{4}\right]-\left(\mathbb{E}\left[w_{m}^{2}\right]\right)^{2} \\
& =R \mathbb{E}\left[\omega_{m}^{4}\right]+\mathbb{E}\left[6 \sum_{i=m}^{N} \sum_{j=m}^{N} \omega_{i}^{2} \omega_{j}^{2}\right]-R^{2} \sigma^{4} \\
& =R \mathbb{E}\left[\omega^{4}\right]+6 \sigma^{4} \mathbb{E}\left[\sum_{i=m}^{N} \sum_{j=m}^{N} 1\right]-R^{2} \sigma^{4}=R \mathbb{E}\left[\omega^{4}\right]+5 R^{2} \sigma^{4} .
\end{aligned}
$$

In that case, the period analog of (14) becomes

$$
\begin{gathered}
\hat{\rho}_{m, \theta_{1}, \theta_{2}}=\frac{\sigma_{m}^{2}-0.5 \alpha_{m}\left[\lambda_{1}+\lambda_{2}\right]+0.25 \beta_{m} \lambda_{1} \lambda_{2}}{\sqrt{\sigma_{m}^{2}-\alpha_{m} \lambda_{1}+0.25 \beta_{m} \lambda_{1}^{2}} \sqrt{\sigma_{m}^{2}-\alpha_{m} \lambda_{2}+0.25 \beta_{m} \lambda_{2}^{2}}} \\
=\frac{\sigma^{2}-0.5 \mathbb{E}\left[\omega^{3}\right]\left[\lambda_{1}+\lambda_{2}\right]+0.25\left[\mathbb{E}\left[\omega^{4}\right]+5 R \sigma^{4}\right] \lambda_{1} \lambda_{2}}{\sqrt{\sigma^{2}-\mathbb{E}\left[\omega^{3}\right] \lambda_{1}+0.25\left(\mathbb{E}\left[\omega^{4}\right]+5 R \sigma^{4}\right) \lambda_{1}^{2}} \sqrt{\sigma^{2}-\mathbb{E}\left[\omega^{3}\right] \lambda_{2}+0.25\left(\mathbb{E}\left[\omega^{4}\right]+5 R \sigma^{4}\right) \lambda_{2}^{2}}} \\
=\frac{4 \sigma^{2}-2 \mathbb{E}\left[\omega^{3}\right]\left[\lambda_{1}+\lambda_{2}\right]+\mathbb{E}\left[\omega^{4}\right] \lambda_{1} \lambda_{2}+5 R \sigma^{4} \lambda_{1} \lambda_{2}}{\sqrt{4 \sigma^{2}-4 \mathbb{E}\left[\omega^{3}\right] \lambda_{1}+\left(\mathbb{E}\left[\omega^{4}\right]+5 R \sigma^{4}\right) \lambda_{1}^{2}} \sqrt{4 \sigma^{2}-4 \mathbb{E}\left[\omega^{3}\right] \lambda_{2}+\left(\mathbb{E}\left[\omega^{4}\right]+5 R \sigma^{4}\right) \lambda_{2}^{2}}} .
\end{gathered}
$$




\section{Appendix B}

Table B-1-1. Test Statistics for Tail Monotonicity (9-year yield record after deleting the best year; Humboldt, Iowa and Brookings, South Dakota)

\begin{tabular}{|c|c|c|c|c|c|c|c|c|c|}
\hline \multicolumn{10}{|c|}{ LTD Statistic (Iowa) } \\
\hline$u / v$ & $1 / 9$ & $2 / 9$ & $3 / 9$ & $4 / 9$ & $5 / 9$ & $6 / 9$ & $7 / 9$ & $8 / 9$ & 1 \\
\hline $1 / 9$ & 0.24 & 0.43 & 0.56 & 0.68 & 0.76 & 0.84 & 0.90 & 0.96 & 1.00 \\
\hline $2 / 9$ & 0.21 & 0.39 & 0.53 & 0.65 & 0.74 & 0.82 & 0.90 & 0.95 & 1.00 \\
\hline $3 / 9$ & 0.19 & 0.35 & 0.49 & 0.62 & 0.72 & 0.81 & 0.89 & 0.95 & 1.00 \\
\hline $4 / 9$ & 0.17 & 0.32 & 0.46 & 0.58 & 0.69 & 0.79 & 0.87 & 0.94 & 1.00 \\
\hline $5 / 9$ & 0.15 & 0.30 & 0.43 & 0.55 & 0.66 & 0.77 & 0.86 & 0.94 & 1.00 \\
\hline $6 / 9$ & 0.14 & 0.27 & 0.40 & 0.52 & 0.64 & 0.74 & 0.84 & 0.93 & 1.00 \\
\hline $7 / 9$ & 0.13 & 0.25 & 0.38 & 0.50 & 0.61 & 0.72 & 0.82 & 0.92 & 1.00 \\
\hline $8 / 9$ & 0.12 & 0.24 & 0.35 & 0.47 & 0.58 & 0.69 & 0.80 & 0.90 & 1.00 \\
\hline 1 & 0.11 & 0.22 & 0.33 & 0.44 & 0.56 & 0.67 & 0.78 & 0.89 & 1.00 \\
\hline \multicolumn{10}{|c|}{ LTD Statistic (South Dakota) } \\
\hline $1 / 9$ & 0.15 & 0.29 & 0.41 & 0.52 & 0.62 & 0.73 & 0.82 & 0.92 & 1.00 \\
\hline $2 / 9$ & 0.14 & 0.27 & 0.40 & 0.51 & 0.62 & 0.72 & 0.82 & 0.91 & 1.00 \\
\hline 3/9 & 0.13 & 0.26 & 0.38 & 0.50 & 0.61 & 0.71 & 0.81 & 0.91 & 1.00 \\
\hline $4 / 9$ & 0.13 & 0.25 & 0.38 & 0.49 & 0.60 & 0.71 & 0.81 & 0.91 & 1.00 \\
\hline $5 / 9$ & 0.12 & 0.24 & 0.36 & 0.48 & 0.59 & 0.70 & 0.80 & 0.90 & 1.00 \\
\hline $6 / 9$ & 0.12 & 0.24 & 0.36 & 0.47 & 0.58 & 0.69 & 0.80 & 0.90 & 1.00 \\
\hline $7 / 9$ & 0.12 & 0.23 & 0.35 & 0.46 & 0.57 & 0.68 & 0.79 & 0.90 & 1.00 \\
\hline $8 / 9$ & 0.11 & 0.23 & 0.34 & 0.45 & 0.56 & 0.67 & 0.78 & 0.89 & 1.00 \\
\hline 1 & 0.11 & 0.22 & 0.33 & 0.44 & 0.56 & 0.67 & 0.78 & 0.89 & 1.00 \\
\hline \multicolumn{10}{|l|}{ (b) } \\
\hline \multicolumn{10}{|c|}{ RTI Statistic (Iowa) } \\
\hline$u / v$ & $1 / 9$ & $2 / 9$ & $3 / 9$ & $4 / 9$ & $5 / 9$ & $6 / 9$ & $7 / 9$ & $8 / 9$ & 1 \\
\hline $1 / 9$ & 1.00 & 0.89 & 0.78 & 0.67 & 0.56 & 0.44 & 0.33 & 0.22 & 0.11 \\
\hline $2 / 9$ & 1.00 & 0.90 & 0.80 & 0.70 & 0.58 & 0.47 & 0.35 & 0.24 & 0.12 \\
\hline $3 / 9$ & 1.00 & 0.92 & 0.83 & 0.72 & 0.61 & 0.50 & 0.38 & 0.26 & 0.13 \\
\hline $4 / 9$ & 1.00 & 0.93 & 0.84 & 0.75 & 0.64 & 0.53 & 0.40 & 0.28 & 0.14 \\
\hline $5 / 9$ & 1.00 & 0.93 & 0.86 & 0.77 & 0.67 & 0.55 & 0.43 & 0.30 & 0.15 \\
\hline $6 / 9$ & 1.00 & 0.94 & 0.87 & 0.79 & 0.69 & 0.58 & 0.46 & 0.32 & 0.17 \\
\hline $7 / 9$ & 1.00 & 0.94 & 0.88 & 0.80 & 0.71 & 0.61 & 0.48 & 0.34 & 0.19 \\
\hline $8 / 9$ & 1.00 & 0.95 & 0.89 & 0.82 & 0.74 & 0.64 & 0.51 & 0.37 & 0.21 \\
\hline 1 & 1.00 & 0.95 & 0.90 & 0.83 & 0.76 & 0.67 & 0.55 & 0.40 & 0.24 \\
\hline \multicolumn{10}{|c|}{ RTI Statistic (South Dakota) } \\
\hline $1 / 9$ & 1.00 & 0.89 & 0.78 & 0.67 & 0.56 & 0.44 & 0.33 & 0.22 & 0.11 \\
\hline $2 / 9$ & 1.00 & 0.89 & 0.79 & 0.68 & 0.57 & 0.47 & 0.34 & 0.23 & 0.11 \\
\hline $3 / 9$ & 1.00 & 0.90 & 0.79 & 0.68 & 0.58 & 0.50 & 0.35 & 0.23 & 0.12 \\
\hline $4 / 9$ & 1.00 & 0.90 & 0.80 & 0.69 & 0.58 & 0.53 & 0.36 & 0.24 & 0.12 \\
\hline $5 / 9$ & 1.00 & 0.90 & 0.80 & 0.70 & 0.59 & 0.55 & 0.36 & 0.25 & 0.13 \\
\hline 6/9 & 1.00 & 0.90 & 0.81 & 0.70 & 0.60 & 0.58 & 0.37 & 0.25 & 0.13 \\
\hline $7 / 9$ & 1.00 & 0.91 & 0.81 & 0.71 & 0.61 & 0.61 & 0.38 & 0.26 & 0.13 \\
\hline 8/9 & 1.00 & 0.91 & 0.82 & 0.72 & 0.62 & 0.64 & 0.39 & 0.27 & 0.14 \\
\hline 1 & 1.00 & 0.91 & 0.82 & 0.73 & 0.63 & 0.67 & 0.40 & 0.27 & 0.14 \\
\hline
\end{tabular}

Note: only mean estimates are reported, standard errors are available upon request. 
Table B-1-2. Test Statistics for Tail Monotonicity (8-year yield record after deleting the best two years; Humboldt, Iowa and Brookings, South Dakota)

\begin{tabular}{|c|c|c|c|c|c|c|c|c|}
\hline \multicolumn{9}{|c|}{ LTD Statistic (Iowa) } \\
\hline$u / v$ & $1 / 8$ & $2 / 8$ & $3 / 8$ & $4 / 8$ & $5 / 8$ & $6 / 8$ & $7 / 8$ & 1 \\
\hline $1 / 8$ & 0.24 & 0.44 & 0.57 & 0.69 & 0.78 & 0.86 & 0.94 & 1.00 \\
\hline $2 / 8$ & 0.22 & 0.41 & 0.54 & 0.67 & 0.77 & 0.85 & 0.93 & 1.00 \\
\hline $3 / 8$ & 0.19 & 0.37 & 0.51 & 0.63 & 0.74 & 0.84 & 0.92 & 1.00 \\
\hline $4 / 8$ & 0.17 & 0.34 & 0.47 & 0.60 & 0.72 & 0.82 & 0.92 & 1.00 \\
\hline $5 / 8$ & 0.16 & 0.31 & 0.45 & 0.58 & 0.70 & 0.81 & 0.91 & 1.00 \\
\hline $6 / 8$ & 0.14 & 0.29 & 0.42 & 0.55 & 0.67 & 0.79 & 0.90 & 1.00 \\
\hline $7 / 8$ & 0.13 & 0.27 & 0.40 & 0.52 & 0.65 & 0.77 & 0.89 & 1.00 \\
\hline 1 & 0.13 & 0.25 & 0.38 & 0.50 & 0.63 & 0.75 & 0.88 & 1.00 \\
\hline \multicolumn{9}{|c|}{ LTD Statistic (South Dakota) } \\
\hline $1 / 8$ & 0.16 & 0.30 & 0.44 & 0.56 & 0.67 & 0.79 & 0.90 & 1.00 \\
\hline $2 / 8$ & 0.15 & 0.29 & 0.42 & 0.55 & 0.66 & 0.79 & 0.90 & 1.00 \\
\hline $3 / 8$ & 0.14 & 0.28 & 0.41 & 0.54 & 0.66 & 0.78 & 0.90 & 1.00 \\
\hline $4 / 8$ & 0.14 & 0.27 & 0.41 & 0.53 & 0.65 & 0.77 & 0.89 & 1.00 \\
\hline $5 / 8$ & 0.13 & 0.27 & 0.40 & 0.52 & 0.64 & 0.77 & 0.89 & 1.00 \\
\hline $6 / 8$ & 0.13 & 0.26 & 0.39 & 0.52 & 0.64 & 0.76 & 0.88 & 1.00 \\
\hline 7/8 & 0.13 & 0.26 & 0.38 & 0.51 & 0.63 & 0.76 & 0.88 & 1.00 \\
\hline 1 & 0.13 & 0.25 & 0.38 & 0.50 & 0.63 & 0.75 & 0.88 & 1.00 \\
\hline
\end{tabular}

\begin{tabular}{ccccccccc} 
(b) \\
\multicolumn{10}{l}{ RTI Statistic (Iowa) } \\
\hline$u / v$ & $1 / 8$ & $2 / 8$ & $3 / 8$ & $4 / 8$ & $5 / 8$ & $6 / 8$ & $7 / 8$ & 1 \\
\hline $1 / 8$ & 1.00 & 0.88 & 0.75 & 0.63 & 0.50 & 0.38 & 0.25 & 0.13 \\
$2 / 8$ & 1.00 & 0.89 & 0.78 & 0.65 & 0.53 & 0.40 & 0.27 & 0.13 \\
$3 / 8$ & 1.00 & 0.91 & 0.80 & 0.68 & 0.56 & 0.42 & 0.28 & 0.14 \\
$4 / 8$ & 1.00 & 0.91 & 0.82 & 0.70 & 0.58 & 0.44 & 0.30 & 0.15 \\
$5 / 8$ & 1.00 & 0.92 & 0.84 & 0.72 & 0.60 & 0.47 & 0.32 & 0.17 \\
$6 / 8$ & 1.00 & 0.93 & 0.85 & 0.74 & 0.63 & 0.49 & 0.34 & 0.18 \\
$7 / 8$ & 1.00 & 0.93 & 0.86 & 0.75 & 0.64 & 0.51 & 0.36 & 0.19 \\
1 & 1.00 & 0.93 & 0.87 & 0.77 & 0.67 & 0.54 & 0.38 & 0.20 \\
\hline RTI Statistic (South Dakota) & & & & & & \\
\hline $1 / 8$ & 1.00 & 0.88 & 0.75 & 0.63 & 0.50 & 0.38 & 0.25 & 0.13 \\
$2 / 8$ & 1.00 & 0.88 & 0.76 & 0.63 & 0.51 & 0.38 & 0.26 & 0.13 \\
$3 / 8$ & 1.00 & 0.88 & 0.76 & 0.64 & 0.52 & 0.39 & 0.26 & 0.13 \\
$4 / 8$ & 1.00 & 0.88 & 0.77 & 0.65 & 0.52 & 0.39 & 0.27 & 0.14 \\
$5 / 8$ & 1.00 & 0.89 & 0.77 & 0.66 & 0.53 & 0.40 & 0.27 & 0.14 \\
$6 / 8$ & 1.00 & 0.89 & 0.78 & 0.66 & 0.54 & 0.41 & 0.28 & 0.14 \\
$7 / 8$ & 1.00 & 0.89 & 0.79 & 0.68 & 0.56 & 0.52 & 0.29 & 0.15 \\
1 & 1.00 & 0.89 & 0.79 & 0.68 & 0.56 & 0.53 & 0.30 & 0.16 \\
\hline
\end{tabular}

Note: only mean estimates are reported, standard errors are available upon request. 
Table B-1-3. Test Statistics for Tail Monotonicity (7-year yield record after deleting the best three years; Humboldt, Iowa and Brookings, South Dakota)

\begin{tabular}{|c|c|c|c|c|c|c|c|}
\hline \multicolumn{8}{|c|}{ LTD Statistic (Iowa) } \\
\hline$u / v$ & $1 / 7$ & $2 / 7$ & $3 / 7$ & $4 / 7$ & $5 / 7$ & $6 / 7$ & 1 \\
\hline $1 / 7$ & 0.26 & 0.46 & 0.61 & 0.73 & 0.83 & 0.92 & 1.00 \\
\hline $2 / 7$ & 0.23 & 0.43 & 0.57 & 0.71 & 0.81 & 0.92 & 1.00 \\
\hline $3 / 7$ & 0.20 & 0.39 & 0.54 & 0.68 & 0.79 & 0.90 & 1.00 \\
\hline $4 / 7$ & 0.18 & 0.36 & 0.51 & 0.65 & 0.77 & 0.89 & 1.00 \\
\hline $5 / 7$ & 0.17 & 0.33 & 0.48 & 0.62 & 0.76 & 0.88 & 1.00 \\
\hline $6 / 7$ & 0.15 & 0.31 & 0.45 & 0.60 & 0.74 & 0.87 & 1.00 \\
\hline 1 & 0.14 & 0.29 & 0.43 & 0.57 & 0.71 & 0.86 & 1.00 \\
\hline \multicolumn{8}{|c|}{ LTD Statistic (South Dakota) } \\
\hline $1 / 7$ & 0.17 & 0.32 & 0.47 & 0.60 & 0.74 & 0.87 & 1.00 \\
\hline $2 / 7$ & 0.15 & 0.31 & 0.46 & 0.60 & 0.73 & 0.87 & 1.00 \\
\hline 3/7 & 0.15 & 0.30 & 0.45 & 0.59 & 0.73 & 0.87 & 1.00 \\
\hline $4 / 7$ & 0.15 & 0.30 & 0.45 & 0.59 & 0.73 & 0.87 & 1.00 \\
\hline $5 / 7$ & 0.14 & 0.29 & 0.44 & 0.58 & 0.72 & 0.86 & 1.00 \\
\hline $6 / 7$ & 0.14 & 0.29 & 0.43 & 0.58 & 0.72 & 0.86 & 1.00 \\
\hline 1 & 0.14 & 0.29 & 0.43 & 0.57 & 0.71 & 0.86 & 1.00 \\
\hline
\end{tabular}

(b)

\begin{tabular}{|c|c|c|c|c|c|c|c|}
\hline \multicolumn{8}{|c|}{ RTI Statistic (Iowa) } \\
\hline$u / v$ & $1 / 7$ & $2 / 7$ & $3 / 7$ & $4 / 7$ & $5 / 7$ & $6 / 7$ & 1 \\
\hline $1 / 7$ & 1.00 & 0.86 & 0.71 & 0.57 & 0.43 & 0.29 & 0.14 \\
\hline $2 / 7$ & 1.00 & 0.88 & 0.74 & 0.60 & 0.46 & 0.30 & 0.15 \\
\hline $3 / 7$ & 1.00 & 0.89 & 0.77 & 0.63 & 0.48 & 0.33 & 0.17 \\
\hline $4 / 7$ & 1.00 & 0.90 & 0.79 & 0.65 & 0.51 & 0.34 & 0.18 \\
\hline $5 / 7$ & 1.00 & 0.91 & 0.81 & 0.67 & 0.53 & 0.37 & 0.19 \\
\hline $6 / 7$ & 1.00 & 0.91 & 0.82 & 0.69 & 0.55 & 0.39 & 0.21 \\
\hline 1 & 1.00 & 0.92 & 0.84 & 0.71 & 0.58 & 0.42 & 0.23 \\
\hline \multicolumn{8}{|c|}{ RTI Statistic (South Dakota) } \\
\hline $1 / 7$ & 1.00 & 0.86 & 0.71 & 0.57 & 0.43 & 0.29 & 0.14 \\
\hline $2 / 7$ & 1.00 & 0.86 & 0.72 & 0.58 & 0.43 & 0.29 & 0.15 \\
\hline 3/7 & 1.00 & 0.86 & 0.72 & 0.58 & 0.44 & 0.29 & 0.15 \\
\hline $4 / 7$ & 1.00 & 0.86 & 0.72 & 0.59 & 0.44 & 0.30 & 0.15 \\
\hline $5 / 7$ & 1.00 & 0.86 & 0.73 & 0.59 & 0.45 & 0.30 & 0.15 \\
\hline $6 / 7$ & 1.00 & 0.86 & 0.73 & 0.60 & 0.46 & 0.31 & 0.16 \\
\hline 1 & 1.00 & 0.86 & 0.73 & 0.61 & 0.47 & 0.32 & 0.17 \\
\hline
\end{tabular}

Note: only mean estimates are reported, standard errors are available upon request. 
Table B-1-4. Test Statistics for Tail Monotonicity (6-year yield record after deleting the best four years; Humboldt, Iowa and Brookings, South Dakota)

(a)

\begin{tabular}{ccccccc}
\hline \multicolumn{2}{l}{ LTD Statistic (Iowa) } & & & & \\
\hline$u / v$ & $1 / 6$ & $2 / 6$ & $3 / 6$ & $4 / 6$ & $5 / 6$ & 1 \\
\hline $1 / 6$ & 0.27 & 0.49 & 0.65 & 0.77 & 0.90 & 1.00 \\
$2 / 6$ & 0.24 & 0.46 & 0.62 & 0.76 & 0.89 & 1.00 \\
$3 / 6$ & 0.22 & 0.42 & 0.59 & 0.74 & 0.88 & 1.00 \\
$4 / 6$ & 0.20 & 0.38 & 0.55 & 0.71 & 0.86 & 1.00 \\
$5 / 6$ & 0.18 & 0.36 & 0.53 & 0.69 & 0.85 & 1.00 \\
1 & 0.17 & 0.33 & 0.50 & 0.67 & 0.83 & 1.00 \\
\hline LTD Statistic (South Dakota) & & & & \\
\hline $1 / 6$ & 0.19 & 0.36 & 0.54 & 0.69 & 0.85 & 1.00 \\
$2 / 6$ & 0.18 & 0.35 & 0.52 & 0.68 & 0.84 & 1.00 \\
$3 / 6$ & 0.17 & 0.35 & 0.52 & 0.68 & 0.84 & 1.00 \\
$4 / 6$ & 0.17 & 0.34 & 0.51 & 0.68 & 0.84 & 1.00 \\
$5 / 6$ & 0.17 & 0.34 & 0.51 & 0.67 & 0.84 & 1.00 \\
1 & 0.17 & 0.33 & 0.50 & 0.67 & 0.83 & 1.00 \\
\hline
\end{tabular}

(b)

\begin{tabular}{|c|c|c|c|c|c|c|}
\hline \multicolumn{7}{|c|}{ RTI Statistic (Iowa) } \\
\hline$u / v$ & $1 / 6$ & $2 / 6$ & $3 / 6$ & $4 / 6$ & $5 / 6$ & 1 \\
\hline $1 / 6$ & 1.00 & 0.83 & 0.67 & 0.50 & 0.33 & 0.17 \\
\hline $2 / 6$ & 1.00 & 0.85 & 0.70 & 0.53 & 0.35 & 0.18 \\
\hline $3 / 6$ & 1.00 & 0.87 & 0.73 & 0.56 & 0.38 & 0.20 \\
\hline $4 / 6$ & 1.00 & 0.88 & 0.75 & 0.59 & 0.40 & 0.21 \\
\hline $5 / 6$ & 1.00 & 0.89 & 0.77 & 0.61 & 0.42 & 0.23 \\
\hline 1 & 1.00 & 0.90 & 0.78 & 0.63 & 0.45 & 0.25 \\
\hline \multicolumn{7}{|c|}{ RTI Statistic (South Dakota) } \\
\hline $1 / 6$ & 1.00 & 0.83 & 0.67 & 0.50 & 0.33 & 0.17 \\
\hline $2 / 6$ & 1.00 & 0.84 & 0.67 & 0.51 & 0.34 & 0.17 \\
\hline $3 / 6$ & 1.00 & 0.84 & 0.68 & 0.51 & 0.34 & 0.17 \\
\hline $4 / 6$ & 1.00 & 0.84 & 0.68 & 0.52 & 0.35 & 0.18 \\
\hline $5 / 6$ & 1.00 & 0.84 & 0.68 & 0.53 & 0.35 & 0.18 \\
\hline 1 & 1.00 & 0.84 & 0.68 & 0.53 & 0.36 & 0.19 \\
\hline
\end{tabular}

Note: only mean estimates are reported, standard errors are available upon request. 
Table B-1-5. Test Statistics for Tail Monotonicity (5-year yield record after deleting the best five years; Humboldt, Iowa and Brookings, South Dakota)

\begin{tabular}{|c|c|c|c|c|c|}
\hline \multicolumn{6}{|c|}{ LTD Statistic (Iowa) } \\
\hline$u / v$ & $1 / 5$ & $2 / 5$ & $3 / 5$ & $4 / 5$ & 1 \\
\hline $1 / 5$ & 0.30 & 0.54 & 0.72 & 0.87 & 1.00 \\
\hline $2 / 5$ & 0.27 & 0.50 & 0.69 & 0.86 & 1.00 \\
\hline $3 / 5$ & 0.24 & 0.46 & 0.66 & 0.84 & 1.00 \\
\hline $4 / 5$ & 0.22 & 0.43 & 0.63 & 0.82 & 1.00 \\
\hline 1 & 0.20 & 0.40 & 0.60 & 0.80 & 1.00 \\
\hline \multicolumn{6}{|c|}{ LTD Statistic (South Dakota) } \\
\hline $1 / 5$ & 0.22 & 0.42 & 0.62 & 0.81 & 1.00 \\
\hline $2 / 5$ & 0.21 & 0.41 & 0.61 & 0.81 & 1.00 \\
\hline $3 / 5$ & 0.20 & 0.41 & 0.61 & 0.81 & 1.00 \\
\hline $4 / 5$ & 0.20 & 0.41 & 0.61 & 0.81 & 1.00 \\
\hline 1 & 0.20 & 0.40 & 0.60 & 0.80 & 1.00 \\
\hline
\end{tabular}

\begin{tabular}{lccccc} 
(b) \\
\hline \multicolumn{7}{l}{ RTI Statistic (Iowa) } \\
\hline$u / v$ & $1 / 5$ & $2 / 5$ & $3 / 5$ & $4 / 5$ & 1 \\
\hline $1 / 5$ & 1.00 & 0.80 & 0.60 & 0.40 & 0.20 \\
$2 / 5$ & 1.00 & 0.83 & 0.63 & 0.43 & 0.22 \\
$3 / 5$ & 1.00 & 0.85 & 0.67 & 0.46 & 0.24 \\
$4 / 5$ & 1.00 & 0.86 & 0.70 & 0.49 & 0.26 \\
1 & 1.00 & 0.87 & 0.72 & 0.52 & 0.29 \\
\hline \multicolumn{7}{l}{ RT Statistic (South Dakota) } & & & \\
\hline $1 / 5$ & 1.00 & 0.80 & 0.60 & 0.40 & 0.20 \\
$2 / 5$ & 1.00 & 0.81 & 0.61 & 0.40 & 0.20 \\
$3 / 5$ & 1.00 & 0.81 & 0.61 & 0.41 & 0.21 \\
$4 / 5$ & 1.00 & 0.80 & 0.61 & 0.42 & 0.21 \\
1 & 1.00 & 0.80 & 0.62 & 0.43 & 0.22 \\
\hline
\end{tabular}

Note: only mean estimates are reported, standard errors are available upon request. 
Table B-1-6. Test Statistics for Tail Monotonicity (4-year yield record after deleting the best six years; Humboldt, Iowa and Brookings, South Dakota)

\begin{tabular}{lcrrr} 
(a) \\
\multicolumn{7}{l}{ LTD Statistic (Iowa) } \\
\hline$u / v$ & $1 / 4$ & $2 / 4$ & $3 / 4$ & 1 \\
\hline $1 / 4$ & 0.33 & 0.61 & 0.80 & 1.00 \\
$2 / 4$ & 0.30 & 0.57 & 0.79 & 1.00 \\
$3 / 4$ & 0.27 & 0.53 & 0.77 & 1.00 \\
1 & 0.25 & 0.50 & 0.75 & 1.00 \\
\hline \multicolumn{4}{l}{ LTD Statistic (South Dakota) } \\
\hline $1 / 4$ & 0.27 & 0.52 & 0.75 & 1.00 \\
$2 / 4$ & 0.26 & 0.51 & 0.75 & 1.00 \\
$3 / 4$ & 0.25 & 0.50 & 0.76 & 1.00 \\
1 & 0.25 & 0.50 & 0.75 & 1.00 \\
\hline
\end{tabular}

\begin{tabular}{lrrrr} 
(b) \\
\multicolumn{7}{l}{ RTI Statistic (Iowa) } \\
\hline$u / v$ & $1 / 4$ & $2 / 4$ & $3 / 4$ & 1 \\
\hline $1 / 4$ & 1.00 & 0.75 & 0.50 & 0.25 \\
$2 / 4$ & 1.00 & 0.78 & 0.54 & 0.27 \\
$3 / 4$ & 1.00 & 0.80 & 0.57 & 0.29 \\
1 & 1.00 & 0.81 & 0.60 & 0.32 \\
\hline \multicolumn{4}{l}{ RTI Statistic (South Dakota) } \\
\hline $1 / 4$ & 1.00 & 0.75 & 0.50 & 0.25 \\
$2 / 4$ & 1.00 & 0.76 & 0.51 & 0.25 \\
$3 / 4$ & 1.00 & 0.76 & 0.51 & 0.25 \\
1 & 1.00 & 0.74 & 0.50 & 0.26 \\
\hline
\end{tabular}

Note: only mean estimates are reported, standard errors are available upon request. 
Table B-2. Kendall's W for Other Counties in Iowa and South Dakota

(a) Floyd, Iowa

\begin{tabular}{lrrrrrrr}
\hline Years of yield record & 10 & 9 & 8 & 7 & 6 & 5 & 4 \\
\hline After deleting years with best yield & 0.33 & 0.27 & 0.19 & 0.12 & 0.09 & 0.08 & 0.07 \\
After deleting years with worst yield & & 0.32 & 0.29 & 0.26 & 0.25 & 0.18 & 0.10 \\
\hline
\end{tabular}

(b) Hamilton, Iowa

\begin{tabular}{lrrrrrrr}
\hline Years of yield record & 10 & 9 & 8 & 7 & 6 & 5 & 4 \\
\hline After deleting years with best yield & 0.36 & 0.31 & 0.24 & 0.17 & 0.10 & 0.06 & 0.06 \\
After deleting years with worst yield & & 0.33 & 0.31 & 0.28 & 0.27 & 0.19 & 0.10 \\
\hline
\end{tabular}

(a) Beadle, South Dakota

\begin{tabular}{lrrrrrrr}
\hline Years of yield record & 10 & 9 & 8 & 7 & 6 & 5 & 4 \\
\hline After deleting years with best yield & 0.53 & 0.52 & 0.51 & 0.52 & 0.54 & 0.52 & 0.51 \\
After deleting years with worst yield & & 0.43 & 0.31 & 0.13 & 0.10 & 0.10 & 0.09 \\
\hline
\end{tabular}

(d) Turner, South Dakota

\begin{tabular}{lrrrrrrr}
\hline Years of yield record & 10 & 9 & 8 & 7 & 6 & 5 & 4 \\
\hline After deleting years with best yield & 0.30 & 0.23 & 0.16 & 0.14 & 0.11 & 0.10 & 0.10 \\
After deleting years with worst yield & & 0.26 & 0.23 & 0.23 & 0.22 & 0.21 & 0.15 \\
\hline
\end{tabular}

(e) Codington, South Dakota

\begin{tabular}{lrrrrrrr}
\hline Years of yield record & 10 & 9 & 8 & 7 & 6 & 5 & 4 \\
\hline After deleting years with best yield & 0.36 & 0.29 & 0.28 & 0.28 & 0.27 & 0.26 & 0.25 \\
After deleting years with worst yield & & 0.14 & 0.13 & 0.10 & 0.10 & 0.08 & 0.04 \\
\hline
\end{tabular}

